\title{
INTRODUCTION AU DROIT DE LA VENTE INTERNATIONALE DE MARCHANDISES
}

\author{
Par Jérôme Huet*
}

\section{Introduction}

On définit volontiers la vente internationale comme une opération ayant "pour effet de réaliser au delà des frontières un flux de marchandises et un reflux de valeurs". ${ }^{1}$ Et l'on considère qu'à ce contrat on ne saurait appliquer en tous points les mêmes règles qu'à la vente interne, car elles ne sont pas toujours propres à satisfaire les exigences du commerce international. ${ }^{2}$ Cela se conçoit aisément.

L'opération est, le plus souvent, conclue entre commerçants - la vente internationale de marchandises est une vente commerciale à l'état pur - et la distance qui les sépare fait qu'elle s'accompagne généralement d'un transport. Il en résulte des risques particuliers pour les

* Agrégé des Facultés de droit. Professeur à l'Université de Paris V-René Descartes. Expert auprès de la CNUDCI.

1 Civ. 1ère, 4 mai 1964, Bull. civ. I, n 228, J. dr. int. (Clunet) 1965. 126, note B. G., achat de blé à une coopérative française et livrable en Allemagne, contrat international, validité d'une clause de paiement par référence à une monnaie étrangère et à son cours au jour du contrat; adde, Civ. 17 mai 1927, DP. 1928. 1. 25, concl. Matter; comp. retenant le critère de la mise en cause des intérêts du commerce international, Civ. 19 février 1930 et 27 janvier 1931, S. 1031. 1. 1, note Niboyet; Civ. 1ère, 18 mai 1971, J. dr. int. (Clunet) 1972. 62, note B. Oppetit, D. 1972. 37, note D. Alexandre; Paris 13 décembre 1975, Rec. crit. dr. int. priv. 1976. 507, note B. Oppetit, Rev. arbitr. 1977. 107, note Ph. Fouchard; Paris 9 novembre 1984, J. dr. int. (Clunet) 1986. 1039, note E. Loquin; et comp. retenant le critère de la diversité des points de rattachements, Civ. 1ère, 4 juillet 1972, J. dr. int. (Clunet) 1972. 843, note B. Oppetit, Rev. crit. dr. int. priv. 1974. 82, note P. Level; et V. V. Heuzé, La vente internationale, $n^{\circ} 4$, très critique sur le sujet.

La Convention de Vienne de 1980 sur la vente internationale de marchandises retient, quant à elle, le fait que les parties ont leur établissement dans des Etats différents, ce qui impliquera le plus souvent un flux et reflux de choses et de monnaie, mais pas toujours: il y a là simplement "une présomption suffisante d'un mouvement de marchandises", B. Audit, La vente internationale, $n^{\circ} 16$.

Adde, sur la question, Ph. Kahn, J.-Cl. Dr. int., Fasc. 565-A-5, Vente commerciale internationale, 1989; Batiffol et Lagarde, T. 2, $n^{\circ}$ 575; et, sur la notion de contrat international, P. Courbe, J.-Cl. Com. Fasc. 335, Contrats commerciaux internationaux, 1986, $\mathrm{n}^{\circ} 5 \mathrm{~s}$.

2 En ce sens, par exemple, Malaurie et Aynès n 65, 272 et 286, qui insistent aussi sur le fait que la diversité des législations nationales entraîne des distorsions de concurrence et l'un des intérêts d'une uniformisation du droit en la matière est de les éliminer. 
marchandises. Et l'éloignement des parties complique aussi les questions de règlement du prix... D'où plusieurs sortes de difficultés: il faut savoir où doit se faire la livraison, qui doit organiser le transport et en supporter le coût, ${ }^{3}$ qui doit assumer les risques d'avarie ou de perte survenue en cours de transport, ${ }^{4}$ quand et comment devra s'effectuer le paiement, ${ }^{5}$ sans compter la nécessité de procéder à toutes sortes de formalités, notamment de dédouanement... Voilà quelques exemples des besoins du commerce international.

Lorsque la vente implique un transport, on parle de "vente maritime", étant donné que pendant longtemps le transport maritime était le plus usité. Consacrant le lien qui unit de cette manière la vente et le transport, une loi du 3 janvier 1969 est venue, en France, codifier les principales règles applicables en pareil cas, et que pour une bonne part la pratique avait de longue date établies: elle offre aux parties de choisir entre la vente "au départ", où la chose passe aux risques et à la charge de l'acheteur aussitôt livrée à quai pour être embarquée, et la vente "à l'arrivée", où elle reste aux risques et à la charge du vendeur jusqu'à ce qu'elle parvienne à destination. ${ }^{6}$

Quant aux règles prévues pour la vente interne, on observe qu'à divers égards elles sont mal adaptées à la vente internationale. Ainsi, pour ne prendre que cet exemple, le Code civil impose au vendeur, dans son art 1609, de délivrer la chose au lieu où elle se trouve au moment du contrat, ce qui signifie qu'il s'en exécutera le plus souvent en la tenant dans ses magasins à la disposition de l'acheteur. ${ }^{7}$ Or, il est rare qu'on retienne cette solution dans le commerce international. ${ }^{8}$ La distance qui sépare les parties impose fréquemment que le vendeur prenne les mesures nécessaires pour l'acheminement des marchandises: il convient, alors, avec l'acheteur qu'il organisera le transport, et parfois même qu'il en assumera le coût. ${ }^{9}$ Et que dire de la règle du droit interne qui fait passer la propriété de la chose, et les

3 Les Incoterms sont volontiers utilisés pour trancher ces difficultés, et V. infra, I, 2.

4 La Convention de Vienne aborde ces questions, et V. sur l'élaboration de ce texte, infra, II, 2.

5 Le crédit documentaire peut être utilisé à ce propos, et V. infra, I, 3.

6 Loi du 3 janvier 1969 relative à l'armement et aux ventes maritimes, art. $31 \mathrm{~s}$ : des ventes au départ, art. $32 \mathrm{~s}$, et des ventes à l'arrivée, art. $36 \mathrm{~s}$, elle distingue en outre la vente $\mathrm{CAF}$, art. $39 \mathrm{~s}$, qui oblige le vendeur à conclure le contrat de transport, à mettre la marchandise à bord et à l'assurer, mais n'en constitue pas moins du point de vue de la charge des risques une vente au départ; et sur ce texte, et les ventes maritimes en général, $\mathrm{V}$. J. CalaisAuloy, Rep. com. Dalloz, $V^{\circ}$ Ventes maritimes, 1974, $\mathrm{n}^{\circ} 3$ et s; $\mathrm{Y}$. Tassel, J.-Cl. Com. Ventes maritimes, Fasc. 1350, 1989, $\mathrm{n}^{\circ} 27$ s; Ripert et Roblot, T. 2, $\mathrm{n}^{\circ} 2251 \mathrm{~s}$; Rodière et du Pontavice, $\mathrm{n}^{\circ} 420 \mathrm{~s}$.

7 Cette règle correspond à la vente "à l'usine" (ou Ex Works), et V. sur cet Incoterm, infra, II, 2.

8 Et V. Y. Tassel, J.-Cl. Com. Ventes maritimes, Fasc. 1385, Vente FAS et FOB,1989, $\mathrm{n}^{\circ} 2$.

9 Ces solutions correspondent à la vente FOB ou CAF, et V. sur ces Incoterms, infra, II, 2. 
risques de perte, sur la tête de l'acheteur dès l'accord des parties. ${ }^{10}$ Certes, de telles dispositions sont supplétives et l'on peut adapter les solutions aux besoins de chacun, mais il n'en reste pas moins que les principes ainsi posés sont souvent loin des besoins existant en pratique. $^{11}$

La dimension commerciale et internationale de l'opération justifie, donc, l'adaptation des règles du droit commun, de caractère national. D'où le travail effectué, depuis longtemps déjà, pour dégager des normes appropriées au sein d'organismes professionnels ou dans le cadre d'institutions comme la Chambre de commerce internationale (C.C.I.). ${ }^{12}$ Dès les années 30 , apparaissent les premières codifications des Incoterms et des usages en matière de crédit documentaire. ${ }^{13}$ La vente étant l'instrument principal des échanges, malgré la concurrence accrue de l'industrie des services, il était logique aussi de voir se développer à son propos un effort d'unification du droit au niveau international: il a trouvé son aboutissement, sous l'égide de la Commission des Nations-Unies pour le droit du commerce international (CNUDCI), ${ }^{14}$ dans la Convention de Vienne sur la vente internationale de marchandises, adoptée en 1980.

Il y a là autant de matériaux pour la constitution de la lex mercatoria, qui est le droit substantiel du commerce international. ${ }^{15}$

Et la Convention de Vienne, qui a été ratifiée par la France, constitue également depuis 1988, date de son entrée en vigueur, notre droit de la vente internationale de marchandises, à côté des règles du Code civil applicables à la vente interne.

L'objectif devait être, donc, de doter la vente internationale de marchandises de règles qui, non seulement, lui soient adaptées, mais, surtout, qui puissent être admises à l'échelle

10 Ou dès l'individualisation de la marchandise par le vendeur s'agissant de choses de genre, art. 1138, al. 2, c. civ.

11 V. à cet égard les développements sur la vente maritime, par Y. Tassel, J.-Cl. Com. Ventes maritimes, Fasc. 1355, Vente CAF, 1989, et surtout, Fasc. 1385, Ventes FAS et FOB,1989.

12 Organisme fondé au début du siècle, et localisé à Paris, 38 Cours Albert 1er, 75008: à cette adresse on peut se procurer les ouvrages réalisés dans le cadre de cette institution, et notamment les Incoterms et les Règles et usances uniformes sur le crédit documentaire.

13 Sur ces deux points, V. infra, I, 2 et 3.

14 Organisme dont le siège est à Vienne, et $\mathrm{V}$. à son sujet et sur sa mise en place, infra $n^{\circ} 15$, en note.

15 V. en ce sens, à propos des réalisations acquises dans le cadre de la CNUDCI, et notamment de la Convention de Vienne, B. Goldman, Les travaux de la CNUDCI, J. dr. int. (Clunet) 1979. 747, p 753. 
mondiale: car seules des règles de droit homogènes, dans les divers pôles commerciaux de la planète, peuvent donner aux échanges internationaux la sécurité juridique souhaitable. ${ }^{16}$

A l'époque où les efforts menés en ce sens en étaient à leurs débuts, on a pu résumer les possibilités d'action en la matière en déclarant que trois sortes d'initiatives pouvaient être prises afin de répondre aux besoins du commerce, et porter remède à l'incertitude du droit applicable: 1) favoriser l'emploi de formes standardisées de contrats comportant des clauses d'arbitrage, pour échapper aux juridictions étatiques, 2) élaborer une codification internationale des règles du droit international privé, fixant la loi compétente en cas de conflit, 3) et, plus radicalement encore, faire disparaître ces sources de conflit de lois en uniformisant le droit substantiel de la vente dans les pays concernés par le commerce international. ${ }^{17}$ En fait, des progrès ont été accomplis sur ces trois terrains.

Dans cette introduction au droit de la vente internationale, il convient de présenter la problématique. On n'abordera, donc, pas le détail de la réglementation donnée à ce contrat par la Convention de Vienne de 1980. On commencera par évoquer le rôle de la pratique dans l'uniformisation du droit en la matière (I), puis on montrera quels efforts ont été faits pour parvenir à mettre en place un droit substantiel de la vente internationale qui soit admis à l'échelle mondiale (II) et, enfin, on donnera des précisions sur le jeu des règles de conflit qui servent à déterminer la loi applicable au contrat, lorsque la solution de difficultés juridiques dépendent encore de cette désignation (III).

\section{Le rôle de la pratique dans l'élaboration du droit de la vente internationale}

\section{A Multiplicité des initiatives}

Sous l'influence des milieux professionnels, des efforts ont été faits pour apporter plus de sécurité dans les relations entre les parties à la vente internationale, et cela de diverses manières: ils ont permis l'élaboration de documents contractuels standardisés $(A)$, la mise au point d'une terminologie normalisée - connue sous le nom d'Incoterms - pour définir les liens entre la vente et le transport des marchandises $(B)$ et l'adoption de règles de crédit documentaire pour assurer, dans les meilleures conditions, le paiement du prix (C).

16 V. B. Audit, La vente internationale, $n^{\circ} 1$; et sur l'uniformisation internationale du droit, J. Hamel, G. Lagarde et H. Jauffret, T. $1, n^{\circ} 43 \mathrm{~s}$.

17 H. C. Gutteridge, L'unification du droit de la vente, Etudes de droit civil dédiées à la mémoire de Henri Capitant, 1938, p 259 s; mais l'auteur notait, en ce qui concerne la troisième possibilité d'action "Nous nous heurtons à l'hostilité très nette des juristes et des commerçants anglais à l'égard de toute mesure qui entraînerait l'abandon ou la modification du Sale of Goods Act de 1893. Qui plus est, il serait impossible à la Grande Bretagne de participer à l'établissement d'un code international qui ne serait pas accepté aussi par le Commonwealth des nations britaniques tout entier et par les Etats-Unis. Il est inadmissible que le degré très appréciable d'unification qui a été réalisé par le Sale of Goods Act et par l'Uniform Law of Sale américaine puisse être mis en péril", op. cit., p 276. 


\section{Développement des contrats-types et des conditions générales de vente}

\section{a) Double origine, double nature}

Parmi les documents contractuels destinés au commerce international, on en distingue deux sortes: les contrats-types, qui émanent généralement d'organismes professionnels, et les conditions générales de vente, qui sont élaborés dans le cadre d'institutions internationales. ${ }^{18}$

S'agissant de contrats-types, celui de la London Corn Trade Association, en vigueur dès la fin du siècle dernier pour ce qui est du commerce de grain, fait figure de précurseur. ${ }^{19}$ L'usage de ce type de document devait se développer au cours du XXème siècle. Ils sont, en principe, spécifiques à une industrie ou un commerce particulier et l'on trouve des textes aussi variés que celui de la Grain and Feed Trade Association, pour le commerce des céréales, ou les conditions générales des fonderies européennes du Comité des associations européennes de fonderie, applicable à cette industrie. ${ }^{20}$ De ces documents ayant trait à la vente, on rapprochera d'autres qui portent sur des opérations voisines, et notamment, pour ce qui est des marchés passés pour l'implantation d'installations industrielles ou commerciales, les conditions-types de la fédération internationale des ingénieurs-conseils, dites conditions "FIDIC". 21

Même si ces modèles sous forme de contrats-types ne tirent jamais leur valeur juridique que de l'adhésion des parties dans la convention qu'elles passent, et donc de la volonté individuelle, on a dit à leur sujet qu'ils tendaient à être l'amorce d'un droit commercial international autonome, ce qui revient à leur attribuer une certaine portée normative. ${ }^{22}$ En tout cas, ces documents sont fort instructifs car ils traitent, en entrant souvent dans le détail, les aspects les plus importants des relations entre vendeur et acheteur: formation du contrat,

18 V. P. Malinverni, Les conditions générales de vente et les contrats-types des chambres syndicales, Thèse L.G.D.J. 1978; A. Meinertzhagen-Limpens, Typologie des conditions générales dans la vente internationale d'objets mobiliers corporels, in "Les ventes internationales de marchandises", 1981, p 79 et s; A. Tunc, Ebauche du droit des contrats professionnels, in "Le droit privé français au milieu du XXème siècle", Etudes Ripert, 1950; P. Padis, La vente commerciale internationale par contrats-types et Incoterms, Gaz. Pal. 1970. 2. doct. 91; A. Seube, Les conditions générales des contrats, in Etudes Jauffrey, 1974, p 621 s; Y. Loussouarn et J.-D. Bredin, $n^{\circ}$ $585 \mathrm{~s}$; J. Thieffry et C. Granier, p $65 \mathrm{~s}$.

19 V. Schwob, Le contrat de la "London corn trade association", Paris,1928; Y. Loussouarn et J.-D. Bredin, $n^{\circ} 562$ s, et 585 s; G. Ripert et R. Roblot, t. 2, n 2546; Ph. Kahn, La vente commerciale internationale, p 726 et s.

20 V. A. Meinertzhagen-Limpens, Typologie des conditions générales, in "Les ventes internationales de marchandises", 1981, p 79 s; P. Malinverni, p 349, où est reproduit le texte relatif à la fonderie.

21 Et V. M. Dubisson, La négociation des marchés internationaux, Feduci, éd. du Moniteur, 1982, p 96 et s; adde, J. Jehl, Le commerce international de la technologie, approche juridique, Thèse, Litec 1985, p 394 et s, à propos du code international de bonne conduite pour le transfert de la technologie, élaboré sous l'égide de la CNUCED. 
qualité de la marchandise et garanties, expédition et prise de livraison, transfert de propriété et charge des risques de perte, conditions de paiement ${ }^{23} \ldots$.

En matière de conditions générales de vente, le mouvement d'unification s'est surtout développé depuis la seconde guerre mondiale. Des experts réunis dans le cadre d'institutions comme la Commission économique pour l'Europe des Nations-Unies (ONU-CEE), où se retrouvent des représentants de pays de l'Est comme de l'Ouest pour travailler à ce que l'on appelle la "facilitation" des échanges internationaux, ont mis en forme des clauses contractuelles à vocation internationale: à titre d'exemple, on peut citer les "Conditions générales pour la fourniture et le montage des matériels d'équipement à l'importation et à l'exportation", un des premiers textes de cet ordre qui fut élaboré dans les années cinquante, ${ }^{24}$ ou encore les "Conditions générales de vente à l'importation et à l'exportation de biens de consommation durables et d'autres produits des industries mécaniques fabriqués en série", dont la rédaction remonte à $1961 .^{25}$ De son côté, le Conseil d'assistance économique mutuelle, ou CAEM, avait établi des textes pour la vente internationale entre les Etats qu'il regroupait, c'est à dire les pays de l'ancien bloc de l'Est, ${ }^{26}$ notamment des conditions générales uniformes pour les contrats de livraison mutuelle de marchandises. ${ }^{27}$

Dans les années 70, un projet prometteur et plus ambitieux, celui de "Conditions générales globales", susceptibles de couvrir toutes sortes de marchandises, a été lancé par la CNUDCI, et certaines clauses qu'il était envisagé d'y voir figurer reprenaient des dispositions déjà contenues dans la Loi uniforme sur la vente internationale, adoptée à La

23 Et V. l'étude approfondie qu'en fait A. Meinèrtzhagen-Limpens, Typologie des conditions générales, in "Les ventes internationales de marchandises", 1981, p $79 \mathrm{~s}$.

24 ONU-CEE $\mathrm{n}^{\circ} 188$ et 574 , et $\mathrm{V}$. la version $188 \mathrm{~A}$ reproduite par B. Oppetit, Droit du commerce international, documents, Thémis 1977, p 363, sous la version 188A, et par P. Malinverni, p 332.

Pour une illustration, V. Civ. 1ère, 26 novembre 1980, J. dr. int. (Clunet) 1981. 355, note Ph. Kahn, livraison et montage d'un silo à grains: l'arrêt écarte les conditions générales de l'ONU-CEE pour les marchés internationaux de fourniture d'équipements à l'exportation - auxquelles s'était référée l'offre du fournisseur faute d'accord exprès entre les parties.

25 ONU-CEE $n^{\circ} 730$.

26 En pratique, le sigle d'origine anglaise, Comecon, était plus volontiers utilisé que celui de CAEM; par suite de l'évolution politique dans les pays de l'ex-Union Soviétique, cet organisme a prononcé sa dissolution en juin 1991, après quarante-deux ans de fonctionnement, V. Le Monde, 29 juin 1991, p 26, "Le Comecon disparaît officiellement.

27 V. J. Rajsky, Le rapprochement et l'unification du droit dans le cadre du conseil d'aide économique et mutuelle, Rev. int. dr. comp. 1976. 461; J. Thieffry et C. Granier, p 58. 
Haye en $1964 .{ }^{28}$ L'intérêt de ces travaux s'est trouvé, cependant, éclipsé par l'adoption en 1980 de la Convention de Vienne sur la vente internationale de marchandises.

\section{b) Différences d'esprit}

Ce qui différencie, sensiblement, les contrats-types et les conditions générales est que les secondes, en raison du fait qu'elles sont élaborées au sein d'organismes publics, recherchent un certain équilibre dans les relations entre les parties, alors que les premiers expriment plus nettement les intérêts corporatistes des professions concernées, et les traduisent en termes juridiques. On a pu s'en offusquer, à l'occasion. Evoquant le caractère supplétif des dispositions de la Loi uniforme de $1964,{ }^{29}$ que l'on retrouve dans la Convention de Vienne, André Tunc souligne les dangers en résultant puisque, de ce fait, les contrats-types des partenaires commerciaux prévalent sur le texte international. ${ }^{30}$

L'opposition entre les deux catégories de documents contractuels, en réalité, n'est cependant pas aussi claire qu'on pourrait être tenté de le croire car il n'est pas rare que les conditions générales consacrent, elles aussi, un certain déséquilibre en faveur d'une des parties. Une illustration peut en être trouvée dans les "Conditions générales de vente à l'importation et à l'exportation de biens de consommation durables et d'autres produits des industries mécaniques fabriqués en série", 31 où l'on voit inscrit nombre de clauses nettement favorables au vendeur: ce texte prévoit un délai de grâce pour le vendeur lors de la livraison (art 6-2), la possibilité de le décharger de sa responsabilité quant à la garantie (sauf la faute lourde, art 9-4), une faculté de choix pour le vendeur quant au moyen qui lui convient le mieux afin de remédier aux vices de la chose (art 9-5), le fait que l'acheteur prend à sa charge les frais et risques du transport s'il doit retourner la chose défectueuse afin de réparation ou de remplacement (art 9-6).

\section{Normalisation des liens entre vente et transport: les Incoterms}

\section{a) Lexique de termes et obligations associées}

Les Incoterms - le mot est une contraction de l'expression "international commerce terms" - constituent un vocabulaire de termes commerciaux normalisés, destiné aux ventes de

28 V. A. Meinertzhagen-Limpens, Typologie des conditions générales, in "Les ventes internationales de marchandises", 1981, p 84; et sur la Loi uniforme de 1964, souvent appelée LUVI, V. infra, II, 1.

29 LUVI, texte précité.

30 Conclusions, in "Les ventes internationales de marchandises", 1981, p 416: et, prenant pour exemple les conditions rédigées par une puissante société sidérurgique où le vendeur était placé dans une position très favorable par rapport à l'acheteur, l'auteur déclare que "ces documents sont rédigés d'une manière parfois scandaleuse" et que "le potentior impose sa volonté". 
marchandises accompagnées d'un transport. Les options possibles sont présentées sous forme de sigles brefs: à chacun d'entre eux, par exemple au sigle EXW, FOB, CIF ou ExShip, ${ }^{32}$ est associée une définition des tâches incombant à chacune des deux parties, de leurs obligations et de leurs charges, en particulier en ce qui concerne le lieu de la délivrance, la fourniture du fret et de l'assurance, l'attribution des risques du transport, la prise de livraison... ${ }^{33}$ Réalisé par la Chambre de commerce internationale, qui a pris l'initiative de la première édition dès 1936, ce lexique est régulièrement actualisé en fonction des besoins existants, et la version actuellement en vigueur date de $1990 .{ }^{34}$

L'objectif est de limiter le plus possible les malentendus et les écarts d'interprétation dans les rapports entre les parties à la vente, ce qui est à l'évidence d'un intérêt primordial. Un autre avantage est que, de cette façon, un lien est assuré de manière claire entre la vente et le transport, de telle sorte que les risques de litiges résultant de la dualité de contrats se trouvent limités. ${ }^{35}$

Il est déclaré, dans l'introduction de la version 1990: "Le but des Incoterms est d'arrêter une série de règles internationales pour l'interprétation des termes commerciaux les plus usités dans le commerce extérieur. On évite ainsi l'incertitude née d'interprétations différentes de ces termes d'un pays à l'autre, ou du moins cette incertitude se trouve considérablement réduite". ${ }^{36}$ Le texte souligne aussi que c'est au cas où les parties se

32 Le terme utilisé doit s'accompagner, dans le cas où c'est nécessaire, du nom du lieu désigné pour les opérations de chargement et de transport: on dit, par exemple, FOB Marseille, ou CAF Bordeaux.

33 Concrètement, ces Incoterms sont présentés dans une brochure, suivant l'ordre croissant des obligations et charges incombant au vendeur, et donc de EXW à DDP, avec pour chaque terme un descriptif en plusieurs points des tâches incombant au vendeur sur la page de gauche, et de celles incombant à l'acheteur sur la page de droite.

V. Incoterms, brochure ICC-CCI ${ }^{\circ} 460,1990$, anglais-français; P. Chauveau, Traité de droit maritime, 1958, $\mathrm{n}^{\circ}$ 902 s; Rodière et du Pontavice, $n^{\circ} 420$ s; F. Eisemann et Y. Derains, La pratique des Incoterms, Jupiter 1988; Loussouarn et Bredin, $\mathrm{n}^{\circ} 588 \mathrm{~s}$; G. Ripert et R. Roblot, T. 2, $\mathrm{n}^{\circ}$ 2546; P. Padis, La consultation pratique en matière de vente commerciale internationale, Gaz. Pal. 1968. 2. doct. 218; du même auteur, La vente commerciale internationale par contrats-types et Incoterms, Gaz. Pal. 1970. 2. doct. 91; J. Calais-Auloy, Rep. com. Dalloz, $V^{\circ}$ Ventes maritimes, 1974; P. Jasinski, Les nouveaux Incoterms et le crédit documentaire, Banque, oct. 1990, p 917 s; V. Heuzé, La vente internationale, $n^{\circ} 251$ s, et 328 s.

34 Après celle de 1936, les versions successives des Incoterms datent de 1953, 1967, 1976, 1980, 1990; et V. Ripert et Roblot, $n^{\circ} 2546$; Y. Loussouarn et J.-D. Bredin, $n^{\circ} 562 \mathrm{~s}$, et 588.

35 Cependant, sur l'autononie du contrat de transport, l'introduction aux Incoterms insiste, soulignant que ce dernier peut apporter des précisions en ce qui concerne les délais ou la prise en charge de certains frais, et ce texte met même en garde sur le fait que les termes commerciaux peuvent y être utilisés dans des sens differents, V. la brochure ICC-CCI ${ }^{\circ} 460$, précitée, introduction, point 15 . Introduction à la brochure ICC-CCI ${ }^{\circ} 460$, précitée, point 1 . 
réfèrent aux Incoterms dans leur contrat, que ceux-ci leur deviennent applicables, ${ }^{37}$ mais il n'est pas interdit d'en admettre également l'application à titre d'usages du commerce international. ${ }^{38}$ En tout état de cause, saisie du texte des Incoterms de 1990 la CNUDCI en a recommandé l'emploi, lors de sa vingt-cinquième session en 1992, reconnaissant qu'ils "constituent une précieuse contribution à la facilitation des échanges internationaux". ${ }^{39}$

La logique des Incoterms, dont l'un des intérêts majeurs est la répartition des risques entre les parties, s'articule autour de la distinction des ventes "au départ" et des ventes "à l'arrivée". ${ }^{40}$ La version de 1953 comptait neuf termes ${ }^{41}$ dont, entre autres, parmi ceux mettant les risques du transport à la charge de l'acheteur, le terme FOB (c'est à dire franco bord, ou free on board) où le vendeur est dit avoir exécuté ses obligations lorsque la marchandise a passé le bastingage du navire au port d'embarquement, ${ }^{42}$ et le terme CAF (contraction de coût assurance fret, ou cost insurance freight) qui réalise un assez bon équilibre entre les intérêts des parties puisque le vendeur, en plus de ce que lui impose le terme FOB, prend en charge les frais d'acheminement de la marchandise jusqu'au lieu de destination convenu, ainsi que l'assurance. ${ }^{43}$ Ce n'en sont pas moins deux modalités de vente "au départ", favorables au vendeur.44

37 "Les commerçants désireux d'utiliser les présentes règles devront spécifier que leurs contrats seront régis par les "Incoterms 1990"", introduction à la brochure ICC-CCI $n^{\circ} 460$, précitée, point 22; aussi bien a-t-on pu assimiler les Incoterms à des contrats-types, V. V. Heuzé, La vente internationale, $n^{\circ} 256$

38 Et V. sur ce point infra, II, 2, (c).

39 Rapport de la session des 2-22 mai 1992, A/47/17, nº 159 à 161.

40 Sur cette distinction, V. J. Calais-Auloy, Rep. com. Dalloz, $V^{\circ}$ Ventes maritimes, 1974, notamment $n^{\circ} 11 ; R$. Rodière et $\mathrm{E}$. du Pontavice, $\mathrm{n}^{\circ} 421 \mathrm{~s}$; la loi du 3 janvier 1969 relative à l'armement et aux ventes maritimes, adopte aussi cette distinction dans ses art. 32 à 38 , et V. supra en introduction du chapitre.

41 Les termes de 1953 étaient: A quai, Franco wagon, FAS, FOB, C et F, CAF, Fret ou port payé jusqu'à, Ex ship, A quai dédouané; et $\mathrm{V}$. Loussouarn et J.-D. Bredin, $\mathrm{n}^{\circ}$ 588; P. Padis, La consultation pratique en matière de vente commerciale internationale, Gaz. Pal. 1968. 2. doct. 218.

42 Et V. sur ce type de vente, M. de Juglart, Les obligations des parties dans la vente FOB, Dr. marit. fr. 1958. 511; Fraikin, Du transfert de propriété dans la vente FOB, Dr. marit. fr. 1950. 107.

43 Sur la vente CAF, désormais appelée CIF dans les Incoterms, et l'équilibre qu'elle réalise, V. notamment J. CalaisAuloy, Rep. com. Dalloz, $V^{\circ}$ Ventes maritimes, 1974, $n^{\circ} 104 \mathrm{~s}$, qui souligne que très tôt, entre 1928 et 1932, on a tenté d'en codifier l'usage dans les "Règles de Varsovie et d'Oxford"; Y. Tassel, J.-Cl. Com. Ventes maritimes, Fasc. 1355, Vente CAF, nature juridique, $1989, n^{\circ} 2$, selon lequel "la vente CAF correspond à un stade avancé du commerce maritime"; adde, M. de Juglart, Le règlement du fret dans la vente CAF, Dr. marit. fr. 1956. 639. La loi du 3 janvier 1969 relative à l'armement et aux ventes maritimes, consacre ses art. 39 à 41 à la vente $\mathrm{CAF}$, et $\mathrm{V}$. sur ce texte, supra en introduction au chapitre.

44 V. R. Rodière et E. du Pontavice, $n^{\circ} 422$ et 425 ; V. Heuzé, La vente internationale, $n^{\circ} 344$.

Il faut distinguer aussi les Incoterms selon les modes de transport visés: certains termes servent exclusivement au transport maritime (transport par mer et par voies navigables intérieures: c'est le cas, par exemple, pour le 
De manière générale, d'ailleurs, les termes figurant dans cette version de 1953 montrent la prédominance qui était celle, à l'époque, des vendeurs: les termes favorables à ces derniers sont les plus nombreux. ${ }^{45}$ Par la suite, les Incoterms "au départ" garderont, certes, une place de choix étant donné que le vendeur souhaite généralement ne pas assumer trop de risques ou de frais, mais on verra se développer les Incoterms "à l'arrivée": ils jouent un rôle accru dans la version de 1990. La raison en est que le poids respectif des vendeurs et acheteurs tend à s'inverser sur l'échiquier marché mondial. ${ }^{46}$ Les Incoterms de 1953 témoignent aussi du fait que le commerce était encore, principalement, maritime. Au gré des révisions ultérieures, de nouveaux sigles vont apparaître pour tenir compte de l'évolution des activités transport et des technologies: il a fallu faire une place à d'autres moyens de locomotion, notamment au transport par avion, ${ }^{47}$ à de nouvelles modalités de fret ("conteneurisation", transport multi-modal...). ${ }^{48}$

La version actuellement en vigueur, qui remonte à 1990, reflète ces transformations. Elle est aussi la première à manifester un souci d'assurer la compatibilité des Incoterms avec le développement, croissant, des échanges de données informatisés (ou EDI), ${ }^{49}$ à quoi l'on doit sans doute rattacher un certain laminage linguistique d'où résulte la disparition du francophone CAF, coût-assurance-fret, au profit de l'anglophone CIF, cost-insurancefreight, subsistant seul désormais pour des besoins d'uniformité.

\section{b) La gamme des Incoterms}

La version de 1990 comporte treize termes, contre quatorze dans celle adoptée dix ans auparavant. ${ }^{50}$ Ces Incoterms, qui tiennent compte du fait que le transport s'effectue par mer ou par d'autres moyens de transport, sont répartis en Incoterms "au départ" et Incoterms "à l'arrivée", et à l'intérieur de cette dualité en quatre catégories, dont les trois premières

terme FOB), et certains à d'autres modes de transport (ainsi le FCA, qui est adapté à tous transports autre que maritime, y compris le transport multi-modal, où il est l'équivalent du terme FOB).

45 V. P. Jasinski, précité, p $917 \mathrm{~s}$.

46 V. P. Jasinski, précité, p 917: "le marché est devenu de plus en plus un marché d'acheteurs".

47 D'où, dans la version de 1980, les FOR, FOT et FOA, qui sont des FOB rail, FOB camion c'est à dire truck, ou FOB aéroport, et qui ont été supprimés en 1990 pour être regroupés au sein du FCA, franco transporteur ou free carrier, qui vise la remise à tout transporteur, $\mathrm{V}$. introduction à la brochure ICC-CCI $n^{\circ} 460$, précitée, point 4 .

48 V. par exemple, à cet égard, le terme FCA, évoqué supra, en note.

49 V. l'introduction à la brochure ICC-CCI ${ }^{\circ} 460$, précitée, points 3 et $18 \mathrm{~s}$; et sur ce type de communication, V. J. Huet, Aspects juridiques de l'EDI, Echanges de Données Informatisés, D. 1991. Chron. 182; Th. Piette-Coudol, L'échange de données informatisées, Gaz. Pal. 1991. Doct. 6-8 oct. 1991.

50 Diminution due à des regroupements, les FOR, FOT et FOA de 1980 (FOB rail, camion ou truck, et aéroport) ayant été réunis, en 1990, dans le FCA, applicable à tous transporteur, et V. sur ce point, supra, en note. 
relèvent des ventes "au départ", et la dernière des ventes "à l'arrivée": $\mathrm{E}$ (un terme), $\mathrm{F}$ (trois termes), C (quatre termes), D (cinq termes). Ils se présentent de la matière suivante.

I Incoterms "au départ", dans lesquels le vendeur assume le moins de coûts et risques résultant du transport, et où figurent: a) le groupe "E", avec un seul terme, 1. Ex W, c'est à dire A l'usine, ex works; b) le groupe "F", où le vendeur se libère par la remise de la chose dans le pays d'exportation et où l'on trouve: 2. FCA, Franco transporteur, free carrier, désignation valable pour tous moyens de transport; 3. FAS, Franco le long du navire, free along side ship, premier des termes propres au transport par mer ou par voies navigables; 4. FOB, Franco bord, free on board, désignation utilisée pour le transport par mer et par voies navigables; $c$ ) le groupe " $C$ " qui réunit: 5 . CFR, Coût et fret, cost and freight, applicable au transport par mer ou voies navigables; 6 . CIF, Coût, assurance et fret (antérieurement CAF en version française), cost, insurance and freight, toujours propre au transport par mer, ou voies navigables; 7. CPT, Port payé jusqu'à, carriage paid to, qui couvre toutes les modalités de transport, ainsi que leur combinaison; 8. CIP, Port payé-assurance comprise-jusqu'à, carriage and insurance paid to, terme qui peut être utilisé pour toutes sortes de transport;

II Incoterms "à l'arrivée" où figure le seul groupe " $\mathrm{D}$ ", dans lequel le vendeur assume le coût et les risques du transport jusqu'au pays d'arrivée: 9. DAF, Rendu frontière, delivered at frontier, valable pour tous modes de transport; 10. DES, Rendu ex ship, delivered ex ship, utilisable seulement pour le transport par mer ou voies navigables; 11. DEQ, Rendu à quai (droits acquittés), delivered ex quay (duty paid), également propre au transport par mer et voies navigables; 12. DDU, Rendu droits non acquittés, delivered duty unpaid, utilisable pour tous modes de transport; 13. DDP, Rendu droits acquittés, delivered duty paid, lui aussi utilisable pour tous modes de transport.

\section{c) Interprétation des Incoterms}

Malgré le succès des Incoterms, il existe néanmoins une ombre au tableau, car les sigles ayant reçu le label de la CCI connaissent, dans d'autres cadres, des définitions parfois un peu différentes: par exemple, celles du code de commerce américain, ${ }^{51}$ ou celles des conditions posées par certaines compagnies maritimes pour le fret international dans leurs conditions générales... 52

Des difficultés risquent donc de surgir lorsque les parties n'auront pas dit à quelle norme il faut se reporter pour définir les termes utilisés par elles. Toutefois, ainsi que la

51 UCC-Sales: les "delivery terms" des sections 2-319 à 2-321; et V. sur ces termes J. M. Stockton, p 99 s.

52 Les "liner terms", et V. D. Le Masson et S. Stenay, Les Incoterms, in "La Convention de Vienne sur la vente internationale et les Incoterms", p 41 s. 
Convention de Vienne y invite, ${ }^{53}$ il sera possible de faire application, pour interpréter la volonté des contractants, des usages du commerce. Et, sur ce fondement, on pourra souvent estimer qu'elles ont entendu se référer aux définitions données aux Incoterms par la Chambre de commerce internationale, puisque l'on tend à considérer qu'elles ont la force d'un usage du commerce international. ${ }^{54}$

Il faut seulement observer, à cet égard, qu'il convient de distinguer parmi les Incoterms selon qu'ils reflètent des habitudes commerciales d'ores et déjà établies, ce qui est le cas pour les plus connus et les plus courants d'entre eux, ou qu'ils ont été créés pour résoudre des problèmes nouveaux, si bien qu'ils constituent, en réalité, une création de la CCI: les premiers, seuls, devraient se voir attribuer une valeur d'usage. ${ }^{55}$

\section{d) Articulation avec la Convention de Vienne}

Pour le reste, il suffit de signaler que l'articulation des Incoterms avec la Convention de Vienne se fait sans peine. ${ }^{56}$ La question se pose en ce qui concerne les dispositions que celle-ci consacre au lieu de livraison des marchandises par le vendeur (art 31 et s) et au moment du transfert à l'acheteur de la charge des risques (art 67 et s). Déjà, le fait que la Convention de Vienne présente un caractère supplétif ${ }^{57}$ supprime, à la racine, tout risque de conflit: l'accord des parties, notamment l'adoption d'un des Incoterms de la CCI, primera toute règle posée par la Convention, qui n'a jamais pour objectif que de trancher les difficultés pouvant survenir en l'absence de volonté exprimée. Comme la pratique montre que ces termes de transport sont très utilisés, il est clair que les règles posées par la Convention

53 Art. 9.

54 V. B. Audit, La vente internationale, $n^{\circ} 82$, note 1 , qui cite en ce sens les sentences CCI $n^{\circ} 80-3130$, J. dr. int. (Clunet ) 1981. 932, obs. Y. Derains, et CCI n 81-3894, J. dr. int. (Clunet) 1982. 987, obs. Y. Derains; dans le même sens, $\mathrm{Ph}$. Fouchard, in "La Convention de Vienne sur la vente internationale et les Incoterms", Rapport de synthèse, $\mathrm{p} 156, \mathrm{n}^{\circ} 16$.

55 V. Y. Derains, Transfert des risques de livraison, in "La Convention de Vienne sur la vente internationale et les Incoterms", p 137: l'auteur souligne que les Incoterms de 1953 doivent se voir reconnaître une valeur d'usage "car à l'époque la CCI a procédé à une consolidation d'usages bien établis", mais qu'il n'en va pas de même pour ceux créés plus tard, et notamment en 1980, leur but n'étant que de "normaliser une pratique désorientée"; V. Heuzé, La vente internationale, $\mathrm{n}^{\circ} 256$, qui souligne l'intérêt d'une référence expresse aux Incoterms de la CCI.

56 La version de 1990 fait référence au nouveau droit de la vente internationale, établi par la Convention de Vienne de 1980, par exemple en ce qui concerne l'emballage des marchandises dont il est prévu par ce texte qu'il incombe au vendeur de l'assurer (art. 35), obligation que l'introduction aux Incoterms déclare tributaire de la connaissance que le vendeur peut avoir de ce que seront les conditions de transport, introduction à la brochure ICC-CCI n ${ }^{\circ} 460$, précitée, point 9.

57 Art. 6 de la Convention. 
en ce qui concerne la livraison et de charge des risques seront souvent précisées, si ce n'est remplacées, par la volonté expresse des parties. ${ }^{58}$

De surcroît, les solutions admises par la Convention de Vienne, pour suppléer le silence des parties, sont en harmonie avec les Incoterms, et la manière dont ils sont utilisés: ainsi, pour une vente impliquant un transport, l'art 31-a retient le principe que la livraison se fait par la remise au premier transporteur pour transmission à l'acheteur, et l'art 67-1 décide que c'est lors de cette remise que s'opère le transfert des risques, ce qui correspond à la philosophie de la vente FOB, la plus usitée en pratique. ${ }^{59}$ Lorsque, à l'inverse, la vente n'implique par un transport, c'est au lieu où le vendeur a son établissement qu'il doit mettre les marchandises à la disposition de l'acheteur, aux termes de l'art 31-c, et c'est au moment où ce dernier vient les retirer que les risques passent à sa charge, selon l'art 69-1: or, la solution correspond à celle de la vente Ex Works. De manière générale, d'ailleurs, il y a comme une affinité de logique entre la Convention de Vienne et le système des Incoterms qui, l'une et l'autre, lient le transfert de risques à la remise de la chose, par le vendeur, à un transporteur ou à l'acquéreur. ${ }^{60}$

\section{Vente et paiement: les règles et usances uniformes pour le crédit documentaire}

\section{a) Délivrance, paiement et crédit}

Outre celui qui existe entre vente et transport, et que permettent d'assurer les Incoterms, il est un autre lien, non moins important, dont il faut tenir compte: le lien entre vente, garantie de paiement et mobilisation de créance. Il est assuré par le biais de ce qu'on appelle le "crédit documentaire".61

58 A moins que ce ne soit par l'effet de l'application d'usages particuliers, et V. en ce sens, B. Audit, La vente internationale, $\mathrm{n}^{\circ} 87$ et 88 .

59 L'art. 32-2 et 3, de la Convention envisage, par ailleurs, que le vendeur soit tenu par le contrat de prendre des dispositions pour le transport, et même qu'il puisse devoir contracter une assurance, ce qui correspond aux ventes CFR, ou CIF.

60 L'art. 31, al. 1er, de la Convention prévoit aussi le cas où les parties ont entendu que la livraison par le vendeur se ferait en un "lieu particulier", ce qui sera souvent le lieu de destination, c'est à dire chez l'acquéreur, et l'art. 69-2 dispose que le transfert des risques s'opère alors par la mise à disposition en ce lieu : ces solutions relèvent de la philosophie des ventes "à l'arrivée", par exemple DDU ou DDP.

61 V. Crédits documentaires, Règles et usances uniformes, CCI n ${ }^{\circ} 400,1984$, révision de 1983, français; et le manuel explicatif, UCP 1974-1983 Revisions compared and explained, Documentary credits, ICC 1984; J. Stoufflet, Rep. com. Dalloz, $V^{\circ}$ Crédit documentaire, 1989; X. Tandeau de Marsac, Le paiement et ses garanties, in Les ventes internationales, 1981, précité, p 391 et s; J.-L. Rives-Lange, Les engagements abstraits pris par le banquier, Banque 1985, p 902 s; P. Jasinski, Les nouveaux Incoterms et le crédit documentaire, Banque, oct. 1990, p 917 s; J.-L. Rives-Lange et M. Contamine-Raynaud, n 636 s; J. Hamel, G. Lagarde et A. Jauffret, T. 2, $1966, \mathrm{n}^{\circ} 1815 \mathrm{~s}$. 
Les exigences pratiques auxquelles répond le crédit documentaire, et qu'explique l'éloignement où se trouvent les parties, peuvent être résumées ainsi: le vendeur souhaite, en principe, avoir toute sécurité quant au règlement du prix par l'acheteur avant de se dessaisir de la marchandise, car les aléas sont nombreux dans le commerce international et les risques d'impayés non négligeables; par ailleurs, il désire souvent pouvoir mobiliser la créance représentant prix, au cas où il a dû consentir des délais de paiement, ce qui n'est pas rare; quant à l'acheteur, il hésite à procéder au paiement avant d'avoir pu vérifier que la marchandise est conforme à la commande, et qu'elle va lui être livrée. Pour trouver une solution, on recourt au crédit documentaire, et c'est dans le contrat de vente que les parties prennent les dispositions nécessaires à cet effet.

Le mécanisme tend à assurer un équilibre entre les intérêts du vendeur, exportateur, auquel il importe d'être payé dès l'embarquement de la marchandise et d'être ainsi protégé contre une insolvabilité ultérieure de son cocontractant, ou contre tous autres aléas, et ceux de l'acheteur, importateur, qui ne souhaite pas règler le prix sans être sûr que son fournisseur a fait le nécessaire pour lui procurer la marchandise convenue et l'acheminer à destination. Afin de satisfaire ces exigences divergentes, on a pris l'habitude de passer par l'intermédiaire d'une ou plusieurs banques pouvant avancer les fonds, et d'utiliser, pour contrôler le bon déroulement des opérations d'exécution, divers documents attestant que la marchandise est en cours de transport. ${ }^{62}$ D'où le nom de crédit "documentaire", adopté par la pratique. L'idée est qu'une banque doit pouvoir effectuer le paiement, pour le compte de l'acheteur et au profit du vendeur, dès lors que ce dernier lui remet les documents énumérés à cet effet au contrat de vente, parmi lesquels figurent un document de transport, qui est généralement un connaissement, ${ }^{63}$ une facture commerciale, éventuellement une police d'assurance. ${ }^{64}$

62 La pratique s'est développée entre les deux guerres à l'instigation des exportateurs américains soucieux d'obtenir des garanties des importateurs européens, en raison des doutes qu'ils avaient sur leur solvabilité, V. J. Hamel, G. Lagarde et A. Jauffret, $\mathrm{n}^{\circ} 1818$.

63 Le transport, en l'occurrence, est le contrat par lequel un transporteur s'oblige, à la demande du vendeur, à acheminer la marchandise à un point de destination particulier, où viendra en prendre livraison l'acheteur, et lors de l'embarquement le transporteur délivre un titre à l'expéditeur, que ce dernier transmet au destinataire afin qu'il puisse prendre possession des marchandises à l'arrivée; un tel document de transport est indispensable au fonctionnement du crédit documentaire, mais ce n'est pas nécessairement un titre représentatif des marchandises, comme est le connaissement: ce peut être un simple bon d'enlèvement, ou autre reçu, délivré par le transporteur, et V. J. Stoufflet, Rép. com. Dalloz, $V^{\circ}$ Crédit documentaire, $\mathrm{n}^{\circ} 15 \mathrm{~s}$.

Le connaissement est prévu en droit interne par l'art. 92, al. 2, c. com., dans le cadre des dispositions sur le gage, où est visée sa fonction de titre représentatif de la marchandise, d'où découle la possibilité de la revendre en cours de transport. 
On conçoit ainsi que le crédit documentaire n'aura généralement lieu d'être utilisé que pour les ventes "au départ", par exemple FOB ou CIF, puisque dans une vente "à l'arrivée" le fournisseur, supportant les risques du transport, ne devrait pas pouvoir prétendre être payé aussitôt qu'il se dessaisit des marchandises, mais seulement une fois l'acheteur entré en leur possession. 65

Là encore, le travail d'harmonisation souhaitable a été réalisé grâce à la Chambre de commerce internationale, à laquelle on doit la mise au point, dès 1933 , d'une première version des "Règles et usances uniformes relatives au crédit documentaire". ${ }^{66}$ Cet ensemble de règles a fait l'objet d'une décision de la CNUDCI, du 6 juillet 1984, laquelle déclare que "les règles et usances uniformes relatives aux crédits documentaires constituent une précieuse contribution à la facilitation des échanges internationaux", et qu'elle en "recommande l'emploi... pour les transactions donnant lieu à l'établissement d'un crédit documentaire". Leur application dans une situation donnée résulte de la référence que les parties on faite à ces règles, mais il est envisageable, comme pour les Incoterms, qu'une valeur d'usage du commerce international doive leur être reconnue. ${ }^{67}$

\section{b) Crédit irrévocable et confirmé}

Le schéma le plus simple et le plus efficace est celui d'une relation à quatre personnages, et d'un crédit irrévocable et confirmé. ${ }^{68}$

Les quatre intervenants, et leurs rôles, sont: 1) l'acheteur, ou donneur d'ordre pour le crédit à consentir parce que c'est sur ses instructions que sa banque doit règler le prix, et l'ordre ainsi donné peut être irrévocable; 2) la banque "apéritrice", celle de l'acheteur, qui s'engage directement envers le vendeur en ouvrant le crédit par l'effet d'une lettre accréditive - simple lettre au demeurant - reflétant son accord avec l'acheteur, et prévoyant les documents à produire pour bénéficier du crédit, une durée du crédit et une échéance pour le paiement; 3) la banque "notificatrice", celle du vendeur, qui a mandat de transmettre la lettre accréditive et de réaliser le crédit pour le compte de la banque de l'acheteur, et qui peut au surplus confirmer ce crédit et s'engager ainsi elle-même; 4) le vendeur, ou bénéficiaire, qui est en cas de crédit irrévocable et confirmé créancier de deux engagements bancaires.

V. en ce sens P. Jasinski, Les nouveaux Incoterms et le crédit documentaire, Banque, oct. 1990, p $917 \mathrm{~s}$; et sur les ventes "au départ", et "à l'arrivée", V. supra, I, 2, (a), les Incoterms.

67 V. en ce sens, M. Vasseur, note sous Com. 14 octobre 1981, D. 1982. 301, qui évoque aussi Trib. com. 8 mars 1976, Rev. jur. com. 1977. 72, note Le Guidec: l'arrêt de la Cour suprême, statuant à propos d'un crédit documentaire irrévocable, citait en visa, à côté de l'art. 1134 c. civ., l'art. 3 des "Règles et usances uniformes". Et, sur cette même question à propos des Incoterms, V. supra, II, 2, (c). 
Dans l'application pratique, lorsque les obligations de livraison du vendeur sont remplies, ce dernier produit les documents convenus à son banquier, qui en opère la vérification sur le plan formel, et doit s'exécuter sans avoir la possibilité de s'y refuser pour des raisons tirées du contrat de vente, ${ }^{69}$ pas plus que pour des motifs liées aux rapports avec le donneur d'ordre. Une fois assuré de l'apparente conformité des documents, le banquier applique les termes de la lettre accréditive, ${ }^{70}$ et selon ce qu'elle dispose, assure par exemple au vendeur le paiement immédiat prévu, ou escompte une lettre de change - appelée alors "traite documentaire" - portant le terme convenu.

Une moindre sécurité est apportée par le crédit irrévocable non confirmé, où le fournisseur n'est créancier d'un engagement que du banquier de l'acheteur, engagement que la banque notificatrice a pour mandat d'exécuter. Elle est encore plus limitée dans le cas d'un crédit révocable, puisque l'acheteur et sa banque peuvent y mettre fin à tout moment. Les règles et usances uniformes prévoient qu'à défaut de précision un crédit doit être considéré comme révocable (art 7).

On le constate ne serait-ce que par ce dernier point, il incombe aux parties à la vente de définir avec précision, dans les clauses du contrat, les dispositions qu'elles souhaitent prendre pour organiser le crédit documentaire convenu: outre le caractère irrévocable ou non du crédit, qui est un aspect essentiel, il faudra qu'elles énumèrent les documents à remettre, l'échéance du paiement... Ensuite, il faut que dans les rapports entre l'acheteur et son banquier ces termes soient repris, afin que puissent être respectées les conditions négociées avec fournisseur, et que ce dernier devra retrouver dans les conditions du crédit consenti. Ce dernier point est important puisque, dans les faits, c'est le fournisseur qui aura posé le crédit documentaire comme une condition de la vente, et donc d'une opération dans laquelle il a voulu ne pas en charge les risques du transport, et désiré pouvoir être assuré du paiement dès l'embarquement des marchandises.

\section{La mise en place d'un droit substantiel de la vente internationale de marchandises}

L'adoption de la Convention de Vienne de 1980 sur la vente internationale de marchandises marque l'aboutissement d'une volonté qui s'est manifestée, tout au long du XXème siècle, de doter ce contrat d'une réglementation uniforme, largement acceptée dans le monde, volonté qui s'est exprimée dans une série de projets et réalisations successifs.

69 V. les RUU, art. 3: "Les crédits sont par leur nature des transactions distinctes des ventes ou autres contrats qui peuvent en former la base", et art. 4; l'explication de cette inopposabilité des exceptions est que la solution contraire, ouvrant la porte à toutes sortes de discussion émanant de l'acheteur, nuirait à l'efficacité du procédé; en droit français, on recourt à la notion de délégation pour fonder cette inopposabilité, V. J. Hamel, G. Lagarde et $A$. Jauffret, $n^{\circ} 1823$; J.-L. Rives-Lange et M. Contamine-Raynaud, $n^{\circ} 718$. 
Les travaux d'Unidroit et de la Conférence de La Haye (A), puis ceux de la CNUDCI qui ont abouti à la Convention de Vienne (B), constituent autant d'étapes dans cette quête d'unité.

\section{A Le projet d'Unidroit et les Conventions de La Haye}

\section{Cent fois sur le métier...}

De longue date, des initiatives ont été prises afin de promouvoir une législation uniforme de la vente internationale. ${ }^{71}$ Entre les deux grandes guerres, un organisme créé à l'instigation du gouvernement italien, l'Institut international de Rome pour l'unification du droit privé, appelé "Unidroit", s'est attelé à cette tâche: en 1935, était établi un projet de texte réglementant la vente de marchandises à transporter. ${ }^{72}$ Transmis à la Société des Nations pour être soumis aux gouvernements susceptibles d'approuver une loi internationale en la matière, il reçut rapidement des réponses positives. ${ }^{73}$

A l'issue de la seconde guerre mondiale, ce travail a été repris dans le cadre de la Conférence de La Haye, et donc sous l'égide du gouvernement néerlandais, qui en assurait le secrétariat. ${ }^{74}$ On décida, par compromis, de scinder en deux aspects les questions à traiter, si bien que ce sont deux conventions, portant lois uniformes, qui furent adoptées en matière de vente, par la conférence internationale réunie à La Haye 1964, et ouvertes à la signature la même année: l'une portait sur la formation du contrat, et l'autre - la plus importante en fait - intitulée convention sur la vente internationale d'objets mobiliers corporels (et volontiers désignée par le sigle LUVI) traitait des effets du contrat. ${ }^{75}$

71 Et V. M. Borysewicz, Conventions et projets de conventions sur la vente internationale de marchandises, in "Les ventes internationales de marchandises", 1981, p 16 et $\mathrm{s}$.

72 Dans les études de droit civil dédiées à la mémoire de Henri Capitant, publiées en 1938, plusieurs articles étaient consacrés aux efforts d'unification du droit de la vente: A. Bagge, Quelques réflexions à propos du transfert des risques dans le projet de loi internationale sur la vente, présenté par l'Institut de Rome pour l'unification du droit privé, p 45 s; P. Chauveau, Un projet de loi internationale sur la vente, p 135 s; H. C. Gutteridge, L'unification du droit de la vente, p 259 s; J. Hamel, Les efforts pour l'unification du droit privé en matière de vente: méthodes et résultats, p $301 \mathrm{~s}$.

73 Une vingtaine en 1937, V. J. Hamel, Les efforts pour l'unification du droit privé en matière de vente, Etudes Capitant, 1938, précitées, p 303: l'auteur souligne que l'Angleterre, toutefois, avait repoussé l'idée d'adhérer à une telle législation uniforme.

74 V. Rev. crit. dr. inter. priv. 1956. 746; G. Ripert et J. Boulanger, $\mathrm{n}^{\circ} 1568$; les travaux de la conférence ont permis d'aboutir, tout d'abord, à une unification des règles de conflit, la Convention de La Haye du 15 juin 1955, portant sur la loi applicable aux ventes internationales d'objet mobiliers, et V. infra, III.

75 Sur ces deux textes, leur genèse et les raisons de leur insuccès, V. Ph. Kahn, J.-Cl. Dr. int., Fasc. 565-A-5, $\mathrm{n}^{\circ} 3$ s; M. Alter, $\mathrm{n}^{\circ} 62 \mathrm{~s} ; \mathrm{J} .-\mathrm{C}$. Boulay, $\mathrm{n}^{\circ} 24$. 
Comme traits marquants de la Convention sur la formation du contrat et de la LUVI, on peut souligner les points suivants: 1) le caractère supplétif des règles posées, 2) l'absence de forme exigée pour le contrat, 3) l'irrévocabilité de l'offre stipulant un délai, 4) l'acceptation du contrat par un acte d'exécution de la commande, 5) la formation de la vente par une acceptation non conforme au cas où l'offrant ne proteste pas et si les modifications ne sont pas substantielles, 6) le transfert des risques à compter de la délivrance, 7) la réalisation de la délivrance par la remise au transporteur lorsque la vente comprend un transport, 8) l'obligation de conformité pesant sur le vendeur et à laquelle il manque si la chose ne possède pas les qualités et particularités prévues, 9) l'obligation de retirer la chose mise à la charge de l'acheteur, 10) les sanctions aménagées en fonction de l'idée de contravention essentielle ou non, 11) la possibilité pour une partie de déclarer unilatéralement le contrat résolu en cas de contravention essentielle... ${ }^{76}$

Ces idées et solutions, pour une part originales, se retrouvent dans la Convention de Vienne de 1980.

\section{Les raisons d'un échec}

Les textes adoptés en 1964, pourtant, n'ont pas connu le succès qu'on pouvait escompter. Ils ne furent pas ratifiés par des pays importants comme les Etats-Unis. Et la France, également, devait se refuser à le faire. A la LUVI, on a reproché, notamment, sa trop grande complexité et le fait ne n'avoir pas suffisamment pris en compte le point de vue de l'ensemble des pays concernés. En particulier, dans cette oeuvre rédigée sur le modèle des systèmes d'Europe continentale, on a dénoncé le fait que le particularisme de systèmes juridiques anglo-américains ne trouvaient pas d'écho. ${ }^{77}$ On lui fit grief, aussi, de présenter un caractère trop théorique et la doctrine américaine s'est montrée fort sévère à son encontre, allant jusqu'à prétendre que le texte ne pourrait pas s'adapter à l'évolution des échanges internationaux. ${ }^{78}$

A dire vrai, dès le début de la conférence internationale de 1964, les Etats-Unis s'étaient prononcés contre le projet, ce qui a lourdement pesé sur le sort du texte. André Tunc raconte les choses en ces termes: "il s'était produit en 1963 quelque chose d'important: c'est que les Etats-Unis entraient à Unidroit. Cela faisait longtemps que certains de leurs juristes leur demandaient de s'intéresser à ce travail d'unification du droit, mais il a fallu attendre le

76 Et V. Ph. Kahn, La convention de La Haye du 1er juillet 1964 portant loi uniforme sur la vente internationale des objets mobiliers corporels, Rev. trim. dr. com. 1964. 689; G. Ripert et R. Roblot, Traité de droit commercial, $1970, \mathrm{n}^{\circ} 2548$.

77 V. E. Bergsten, Le rôle de la convention des Nations-Unies, in "Le nouveau droit de la vente internationale", 1987, p 1247.

78 V. A. Farnsworth, "Unification of sales law at the regional and international level, Why they behave like americans?", in Aspects of comparative commercial law, Université McGill 1969, P. 110 et s. 
dernier moment pour qu'ils le fassent. Ils ont tout de suite demandé que l'on suspende la tenue de la conférence de La Haye... Bien sûr, leur absence jusque là était fâcheuse. La requête a pourtant été rejetée par le gouvernement néerlandais. Je ne suis pour rien dans la décision... Mais je l'approuvais entièrement. La Conférence devait se réunir au mois d'avril, toutes les convocations avaient été adressées, le texte avait connu plusieurs versions successives qu'on communiquait chaque fois aux gouvernements. Il serait devenu un serpent de mer si l'on avait repris le travail... Mais, bien sûr, le travail de la Conférence diplomatique qui allait se réunir était faussé dès le départ".79

Il faut rappeler que les Etats-Unis, à l'époque, travaillaient, chez eux, à la rédaction d'un Code de commerce uniforme dont l'article 2, consacré à la vente, devait être une des pièces maîtresses, et ils ne s'étaient sans doute pas sentis prêts à participer à une réflexion internationale sur le sujet avant 1952, date où le première état de sa rédaction a été arrêté, ou même avant 1962 qui est l'année où l'Etat de New York l'a finalement adopté, constituant alors le quinzième Etat américain à le faire. ${ }^{80}$

Quant à la France, elle n'a pas manifesté on plus un grand enthousiasme pour les résultats obtenus alors même qu'elle avait joué un rôle majeur dans les travaux, notamment par la présence du Doyen Hamel et le rôle de secrétaire tenu par André Tunc. Ce dernier raconte que "le garde des sceaux a été immédiatement hostile à ce texte", reprochant aux juristes français d'avoir "tout abandonné aux Anglo-Saxons". 81 Il paraît qu'il fallut développer toute "une action diplomatique pour que... la France signe la convention à la dernière minute". 82

\section{B L'institution de la CNUDCI et la Convention de Vienne}

\section{Le plus large consensus}

En 1966 lorsque la CNUDCI a été fondée, ${ }^{83}$ et en 1968 quand se sont ouverts ses travaux, on pouvait constater le peu d'adhésions aux Conventions de La Haye. Il fut, donc,

79 Et d'ajouter, à propos de "l'attitude de la délégation américaine", lors de la conférence: "Tout de suite elle a fait savoir qu'il n'était pas question pour elle de ratifier le texte de La Haye quand il serait voté", A. Tunc, Conclusions, in "Les ventes internationales de marchandises", 1981, p 410 et s.

80 Aujourd'hui, le code de commerce uniforme américain a été adopté, avec des variantes, par quarante-neuf des cinquante Etats des Etats-Unis, V. J. J. White et R. S. Summers, p 5; adde, sur ce texte, les références données in fine.

81 A. Tunc, Conclusions, in "Les ventes internationales de marchandises", 1981, p 413.

82 A. Tunc, Conclusions, in "Les ventes internationales de marchandises", 1981, p 413.

83 La CNUDCI, Commission des Nations-Unies pour le Droit Commercial International (en anglais UNCITRAL), a été créée par l'Assemblée générale de l'ONU en 1966 et répond au souci, figurant dans la Charte de l'Organisation, d'améliorer le fonctionnement du commerce international par la codification du droit: trente-six Etats, représentatifs des différentes tendances juridiques dans le monde, et diverses institutions, participent à ses 
aussitôt envisagé de reprendre le sujet de la vente internationale, avec l'intention de parvenir à une convention susceptible de rencontrer un large consensus. Un des progrès réalisés devait être de traiter dans un seul texte les questions de formation et d'exécution de la vente, alors qu'elles était dispersées en 1964 dans deux conventions distinctes.

Une Conférence internationale fut convoquée lorsque le projet eut reçu l'approbation de l'Assemblée générale des Nations-Unies: elle allait réunir soixante-deux Etats et huit organisations internationales, à Vienne, du 10 mars au 18 avril 1980, pour en adopter le texte. ${ }^{84}$ La Convention de Vienne sur la vente internationale de marchandises, parfois désignée sous le sigle CVIM, est entrée en vigueur le 1er janvier 1988, dans les délais prévus, après avoir été l'objet de sa dixième ratification. La France l'avait, quant à elle, ratifiée dès 1982, sans avoir eu à dénoncer son adhésion aux Conventions de La Haye qu'elle n'avait jamais adoptées. ${ }^{85}$

Depuis cette ratification, et l'entrée en application de la Convention, il existe désormais, en droit français, deux régimes juridiques pour la vente de marchandises: d'un côté, les règles du Code civil et du droit commercial applicables aux ventes internes, et, de l'autre, le régime de la Convention qui a vocation à couvrir les ventes ayant une dimension internationale. ${ }^{86}$ Par opposition avec le droit interne, la réglementation de la vente résultant de la Convention tend à répondre, avec un certain pragmatisme, aux besoins du commerce international.

Il faut seulement prendre garde ce que ce nouveau droit de la vente internationale a une portée qui reste limitée, car il règle pas toutes les questions que les relations entre les parties peuvent poser, et notamment celles touchant à la validité du contrat et au transfert de

travaux, et V. Y. Daudet et L. Dubouis, J.-Cl. dr. int., Fasc. 13-3, La coutume, codification, 1991, $\mathrm{n}^{\circ} 27 \mathrm{~s}$ (qui rappellent que la création de la CNUDCI répond à la nécessité de disposer de "lois bien conçues et modernes propres à assurer l'égalité dans les échanges commerciaux internationaux", résolution de l'Assemblée générale de Nations-Unies, 17 décembre 1966); Z. Haquani, J.-Cl. dr. int., Fasc. 131, Organismes internationaux en matière de production, de commerce et de développement, 1992, $\mathrm{n}^{\circ} 34 \mathrm{~s}$; adde, B. Goldman, Les travaux de la CNUDCI, J. dr. int. (Clunet) 1979, 753.

84 Parallèlement avait été adopté, puis devait être modifié en fonction des termes de la Convention de Vienne, un texte sur la prescription en matière de vente internationale de marchandises, la Convention de New-York de 1974, V. B. Audit, La vente internationale, précité, $n^{\circ}$ I, A, 1, (a).

85 Loi du 18 juillet 1982; le texte a été publié au JO du 27 décembre 1987, p 15241, D. 1988. L. 30; et V. R. Houin et M. Pédamon, $n^{\circ} 563$.

Pour être à même de ratifier la Convention de Vienne, il faut pour les Etats qui ont adopté les Conventions de La Haye de 1964, ou l'une d'entre elles, dénoncer préalablement cette adhésion, art. 99-3 de la Convention de Vienne.

86 V. B. Audit, La vente internationale, $n^{\circ} 15$, qui montre l'intérêt accru que cela donne à la définition de la vente internationale; $\mathrm{Cl}$. Witz, L'exclusion de la Convention des Nations-Unies sur les contrats de vente internationale de marchandises par la volonté des parties, D. 1990. Chron. 107, in limine. 
propriété. ${ }^{87}$ Aussi bien, alors même que la Convention de Vienne doit s'appliquer, le droit interne, désigné par les règles de conflits de lois, a vocation à relayer le droit uniforme. ${ }^{88}$

\section{Esprit et organisation la Convention}

$\mathrm{Si}$, dans la terminologie, pour montrer le passage du dogmatisme de 1964 au réalisme qui devait s'imposer, on a remplacé les termes "objets mobiliers corporels" par celui de "marchandises et troqué le mot "délivrance" pour celui de "livraison", 89 il est clair que sur le fond il $\mathrm{y}$ a de très nombreux points communs entre la Convention de Vienne, d'une part, la LUVI et la loi uniforme sur la formation du contrat de vente, adoptées à La Haye, d'autre part. Il faut dire que les lois adoptées en 1964 ont servi de base à partir de quoi les experts de la CNUDCI ont travaillé, de manière à pouvoir aboutir dans un délai convenable.

Il n'en demeure pas moins que le texte de 1980 est plus clair et mieux organisé que ceux de 1964 , et, quant à la méthode, il n'est pas niable que le processus d'élaboration et d'adoption de la Convention de Vienne a permis à celle-ci de rassembler des énergies venant d'horizons les plus divers et, donc, de démontrer son éclectisme, ce qui a grandement favorisé l'engouement pour le texte nouveau. La liste des onze premiers Etats l'ayant ratifié l'illustre bien: elle compte l'Argentine, la Chine, l'Egypte, les Etats-Unis, la France, la Hongrie, l'Italie, le Lesotho, la Syrie, la Yougoslavie, la Zambie, qui sont représentatifs aussi bien des pays développés que des pays en voie de développement, des pays d'économie libérale que d'économie dirigée, des pays de Common Law que des pays de tradition romano-germanique, et qui se trouvent répartis sur tous les continents. ${ }^{90}$ Aujourd'hui, la Convention compte plus d'une trentaine d'adhérents. ${ }^{91}$

Quant à ses modalités de ratification, la Convention de Vienne reste marquée par l'héritage des textes de La Haye. Il est, en effet, prévu qu'un pays peut n'adhérer à la Convention qu'en partie, en limitant son adoption à l'un des deux aspects que sont la formation et les effets du contrat. Plus précisément, la ratification par un Etat porte toujours, et nécessairement, d'une part, sur la première partie du texte (champ d'application

87 Art. 4 de la Convention.

88 V. sur ce point, III.

89 V. M. Borysewicz, Conventions et projets de convention sur la vente internationale de marchandises, in "La vente internationale de marchandises", 1981, p $46 \mathrm{~s}$.

90 V. E. Bergsten, Le rôle de la convention des Nations-Unies, in "Le nouveau droit de la vente internationale", 1987, p 1248

91 V. V. Heuzé, La vente internationale, p 407, qui en dénombre trente-quatre en 1992: Argentine, Australie, Autriche, Biélorussie, Bulgarie, Canada, Chili, Chine populaire, Danemark, Egypte, Equateur, Espagne, EtatsUnis, Finlande, France, Hongrie, Irak, Italie, Lesotho, Mexique, Norvège, Ouganda, Pays-Bas, RDA, RFA, Roumanie, Suède, Suisse, Syrie, Tchécoslovaquie, Ukraine, URSS, Yougoslavie, Zambie (on note l'absence de la Grande-Bretagne et du Japon). 
et dispositions générales) et sur la quatrième partie (dispositions finales), et, d'autre part, sur l'une ou l'autre - ou l'une et l'autre - des deuxième (formation du contrat) et troisième parties (effets du contrat), l'art 92 de la Convention prévoyant qu'il est possible de ne pas adopter soit la deuxième, soit la troisième. ${ }^{92}$ Historiquement, cette possibilité de choix est ce qui subsiste de la dualité d'approche admise à La Haye en 1964 et l'on voit, par là, se confirmer la difficulté de réunir en un seul instrument l'ensemble des dispositions relatives à la vente. On a pu regretter la possibilité d'une telle ratification partielle et souligner que cela risquerait de "porter atteinte à l'uniformisation souhaitable des règles" en cause. ${ }^{93}$

Pour le reste, il faut souligner que la Convention de Vienne prévoit peu de réserves, ouvertes aux Etats contractants. ${ }^{94}$ Une première autorise un pays à déclarer qu'il n'entend appliquer le texte que dans les rapports avec un autre Etat ayant adhéré à la Convention (art 95). ${ }^{95}$ Une deuxième a trait à la forme de la vente internationale: si la Convention pose en principe que le contrat est purement consensuel (art 11), elle admet qu'un Etat puisse imposer à ses ressortissants de le passer selon une forme particulière (art 12 et 96). ${ }^{96} \mathrm{Et}$ la troisième permet à des Etats contractants, s'ils en font la déclaration préalable, de décider d'appliquer dans leurs rapports réciproques des règles particulières, différentes de celles de la Convention (art 94). 97

\section{Comparaison avec le droit français interne}

Il est intéressant de rechercher ce qui fait le particularisme du régime de la vente internationale, issu de la Convention de Vienne, par rapport au droit français applicable à la vente interne. Certes, à bien des points de vue, les solutions retenues ici et là sont

92 Peu de pays ont choisi de n'adhérer, ainsi, que partiellement à la Convention: à ce jour, le Danemark, la Finlande, la Norvège et la Suède l'on fait, limitant leur adhésion à la partie relative à la formation du contrat, et V. V. Heuzé, La vente internationale, p 407.

93 I. Fadlallah, Rev. int. dr. comp. 1987. 292, présentation de l'ouvrage de l'Institut suisse de droit comparé sur la convention de Vienne, précité.

94 Elles figurent aux art. 95 s, et l'art. 98 prévoit qu'aucune autre réserve n'est autorisée; à cela s'ajoute néanmoins, comme une sorte de réserve implicite, la liberté que donne l'art. 28 de la Convention en laissant latitude aux juges saisi d'une difficulté d'exécution de la vente de ne pas ordonner l'exécution en nature, s'il ne le ferait pas en vertu de son propre droit, et V. sur ce point infra, \$3, Exécution en nature.

Ce texte permet de déroger à l'art. 1-1, b.

96 Les art. 12 et 96 de la Convention permettent à un Etat contractant de déroger aux art. 11 et 29 lorsque sa législation exige que les contrats soient conclus par écrit, et V. B. Audit, La vente internationale, $n^{\circ} 75, q^{4}$ explique cette possibilité de réserve par le fait que certains pays, à économie dirigée ou planifiée, exigent une forme pour la vente internationale: ont utilisé cette réserve, i'Argentine, la Biélorussie, la Bulgarie, le Chili, la Hongrie et l'Ukraine, V. V. Heuzé, La vente internationale, p 407.

97 Le Danemark, la Finlande, la Norvège et la Suède l'on fait, dans leurs relations respectives, et V. V. Heuzé, La vente internationale, p 407. 
voisines, voire identiques. Mais les différences, où même les divergences, n'en sont pas moins réelles. ${ }^{98}$

On peut chercher à les dénombrer. Les plus notables tiennent à la présence de dispositions d'inspiration anglo-américaine: aux termes de la Convention, la formation du contrat peut être admise, parfois, malgré le défaut de concordance entre l'offre et l'acceptation (art 19); 99 l'efficacité du contrat semble reconnue quand bien même le prix des marchandises est indéterminé (art 55); ${ }^{100}$ le vendeur qui a mal exécuté ses obligations dispose du droit d'y "porter remède", même après l'échéance (art 48); ${ }^{101}$ le contractant victime d'une mauvaise exécution doit faire son possible pour limiter l'ampleur du dommage (art 77)...102 On voit intervenir aussi l'idée très pragmatique, reprise de la "detrimental reliance" de la Common $\mathrm{Law}_{1}{ }^{103}$ consistant à faire produire effet à une situation de fait, à l'encontre de celui qui l'a créée, lorsqu'une autre personne s'est fondée sur la confiance inspirée par cette situation: on l'observe en ce qui concerne l'offre de contracter, s'agissant de la rendre irrévocable $\left(\operatorname{art~16)},{ }^{104}\right.$ et à propos de la modification du contrat (art 29). ${ }^{105}$

Mais bien des différence dénotent, simplement, le souci de retenir des solution adaptées aux besoins du commerce: ainsi, dans la Convention, les raisons que l'acheteur peut avoir de critiquer la marchandise livrée relèvent d'une seule et même notion, le défaut de conformité (art 35), à laquelle s'applique un régime unique marqué par le délai deux ans, à compter de la remise de la chose, pendant lequel l'acheteur est en droit de le dénoncer (art 39); et d'autres solutions comme l'impossibilité pour un juge d'octroyer un délai de grâce (art 45 et 61), ou encore le droit pour l'acheteur de procéder un achat de remplacement et celui pour le

98 Et V. Mouly, Que change la Convention de Vienne sur la vente internationale par rapport au droit français interne?, D. 1991. Chron. 78.

Cette question est désignée dans la doctrine américaine par la formule de "battle of the forms".

100 Sur ce point, toutefois, il y a une ambiguitté dans la convention, étant donné que l'art. 14 semble imposer le contraire.

101 C'est le "seller's right to cure" du code de commerce uniforme américain.

102 C'est le "duty to mitigate" du droit commun des contrats anglo-américain.

103 La théorie de la "detrimental reliance, ou "promissory estoppel", est une source d'obligations complémentaire de celle que constitue le contrat: selon ce principe, une obligation naît au profit de toute personne ayant agi à son "détriment", parce qu'elle a fait "confiance" à un état de choses, le débiteur de l'obligation étant la personne qui a fait se développer ce sentiment de confiance, et laissé faire les choses; et V. T. Galligan, Le droit des contrats, in Droit des Etats-Unis, $n^{\circ} 132$.

104 Elle devient irrévocable s'il était raisonnable de la considérer telle et que son destinataire a agi en conséquence.

105 L'exigence d'un écrit pour toute modification du contrat peut être levée si une partie s'est fondée sur le comportement de l'autre, et V. Ch. Mouly, Que change la Convention de Vienne sur la vente internationale par rapport au droit français interne?, D. 1991. Chron. 78. 
vendeur de procéder à une vente compensatoire (art 75 et 76), s'inspirent de la même philosophie.

De manière générale, une des constantes de la Convention est son pragmatisme. Il se manifeste, en particulier, dans le souci de donner effet au contrat, voire de le sauver, autant que faire se peut: que se soit au moment de sa conclusion (défaut de concordance entre l'acceptation et l'offre, art 19, précité), ou lors de son exécution (obligation de donner un délai avant de procéder à la résolution, art 49 et 64 ; droit de "porter remède" accordé au vendeur, art 48 , précité). Et surtout, toujours dans cette optique pragmatique, la Convention de Vienne cherche à éviter que les contractants ne doivent s'en remettre à la justice pour trancher leurs différends, obtenir satisfaction, ou trouver une issue. D'où les voies de droit unilatérales, ouvertes à l'une ou l'autre des parties, comme la possibilité qui leur est offerte dans certaines circonstances prononcer la résolution du contrat: que ce soit l'acheteur insatisfait (art 49), ${ }^{106}$ ou le vendeur impayé (art 64). Or, cela va directement à l'encontre de la volonté du Code civil de soumettre le contrat, son exécution et son dénouement, au contrôle du juge, afin d'éviter l'abus de puissance d'une partie sur l'autre, ou les décisions individuelles trop rapidement prises (art 1184). Mais il est vrai qu'en matière d'échanges internationaux le recours à la justice est beaucoup plus pesant encore que dans l'ordre interne.

\section{Les conflits de lois en matière de vente internationale de marchandises}

\section{A Rôle des règles de conflit}

Malgré l'uniformisation du droit substantiel de la vente internationale réalisée par la Convention de Vienne de 1980, un rôle important reste dévolu aux règles de conflit de lois. ${ }^{107}$ Et cela, de deux manières:

D'une part, en effet, la Convention prévoit elle-même qu'à titre complémentaire, elle est applicable à un contrat de vente internationale, quand bien même les parties n'ont pas leur établissement dans des Etats contractants, à partir du moment où les règles de droit

106 L'acheteur peut déclarer le contrat résolu par suite de l'inexécution d'une des ses obligations par le vendeur, pour autant qu'elle constitue une contravention essentielle au contrat; il peut également prendre, de lui-même, une mesure de dédommagement, par exemple procéder à une réfaction du prix, art. 50.

107 Même lorsque la Convention de Vienne est applicable, et qu'on se trouve ainsi dans le cadre du droit substantiel unifié de la vente internationale, il peut demeurer nécessaire de faire jouer les règles de conflit de lois: pour connaître la loi dont dépend l'interprétation, nationale, à donner aux termes de la Convention; de fait, on admet qu'en matière de législation uniforme, et en l'absence d'instance juridictionnelle supra-nationale chargée de l'interpréter, c'est à l'interprétation nationale qu'il faut se référer, et donc à l'interprétation donnée par les juridictions de l'ordre juridique déclarée compétent par le droit international privé, V. en ce sens, l'arrêt Hocke, Com. 4 mars 1963, JCP. 1963. II. 13376, note P. Lescot, J. dr. int. (Clunet) 1964. 806, note B. Goldman, Rev. crit. dr. int. priv. 1964. 235, chron. P. Lagarde, rendu en matière de lettre de change; et V. sur le sujet, notamment, P. Mayer, $\mathrm{n}^{\circ} 94 \mathrm{~s}$. 
international privé désignent comme compétent le droit national d'un Etat ayant adopté cette Convention. ${ }^{108}$ Les règles de conflit ramènent alors au droit uniforme de la vente internationale.

D'autre part, il faut rechercher le droit national applicable au contrat, et s'appuyer à cet effet sur les règles de conflit, soit lorsque se posent des questions qui ne sont pas traitées par la Convention, notamment celles de la validité du contrat et du transfert de propriété, ${ }^{109}$ soit tout simplement parce que la Convention de Vienne n'est pas applicable faute, notamment, pour les Etats où les parties ont leur établissement d'avoir ratifié ce texte. ${ }^{110}$

Force est donc, dans ces derniers cas, d'en revenir au droit international privé classique. D'où l'intérêt de se demander si l'effort d'unification du droit de la vente internationale s'est manifesté sur ce terrain également.

\section{B Convention de La Haye de 1955}

L'uniformisation qui existe en la matière est due à la Conférence de La Haye. Avant même d'aboutir à une première unification des règles substantielles de la vente internationale, ${ }^{111}$ ses travaux avaient permis d'opérer une unification des règles de conflit.

De fait, la Convention de La Haye du 15 juin 1955 sur la loi applicable aux ventes internationales d'objet mobiliers, entrée en vigueur en 1964, a posé des règles communes pour la solution des conflits de lois en la matière. ${ }^{112}$ La France est un des pays l'ayant ratifiée. ${ }^{113}$ Comme elle est dotée d'une portée dite "universelle", elle tient lieu de droit

Art. 1, b de la Convention.

109 Art. 4 de la Convention de Vienne; la loi désignée par les règles de conflit s'appliquera, de même, aux questions d'encadrement du contrat, par exemple à la répression du refus de vente.

Au demeurant, l'art. $7-2^{\circ}$ de la Convention de Vienne renvoie expressément à la loi désignée par les règles du droit international privé lorsque, s'agissant de questions concernant les matières qu'elle régit, on ne trouve pas de réponse dans les règles qu'elle formule, ni dans les principes généraux dont elle s'inspire.

110 Art. 1, a.

111 V. sur ce point supra, II, A.

112 V. G. Ripert et R. Roblot, T. 2, $n^{\circ}$ 2545-2; Y. Loussouarn et P. Bourel, $\mathrm{n}^{\circ}$ 378; P. Mayer, $\mathrm{n}^{\circ}$ 727; B. Audit, Droit international privé, $\mathrm{n}^{\circ}$ 792; Ph. Kahn, J.-Cl. Dr. int. Fasc. 565-A-5, $\mathrm{n}^{\circ} 157 \mathrm{~s}$; V. Heuzé, La vente internationale, $\mathrm{n}^{\circ} 16$, et $18 \mathrm{~s}$, avec ce texte reproduit p $373 \mathrm{~s}$; adde, J. Thieffry et C. Granier, p $199 \mathrm{~s}$.

L'adoption, dans le cadre de la Communauté européenne, de la Convention de Rome de 1980 sur la loi applicable aux obligations contractuelles n'a pas porté atteinte, en matière de vente de marchandises, au jeu de la Convention de La Haye, pour les pays l'ayant ratifiée: outre le principe specialia generalibus derogant, la Convention de Rome elle-même impose cette solution (art. 21).

113 Cette Convention a été ratifiée par neuf Etats, dont la France (avec la Belgique, le Danemark, la Finlande, l'Italie, le Niger, la Norvège, la Suède et la Suisse), et V. V. Heuzé, La vente internationale, p 373; adde, B. Audit, La vente internationale, $n^{\circ} 1$. 
international privé pour ceux qui l'ont adoptée, et s'applique donc sans condition de réciprocité et sans tenir compte de ce que la loi désignée est celle d'un Etat qui en fait, ou non, partie. C'est, en France, à ce texte qu'il faut se référer pour connaître les règles de conflit de lois applicables à la vente de marchandises. ${ }^{114}$ Cette Convention retient comme loi compétente celle que les parties au contrat ont choisie ${ }^{115}$ et, à défaut, celle du pays où le vendeur a sa résidence habituelle au moment où il reçoit la commande, ou celle du pays où l'acheteur a sa résidence habituelle si c'est dans ce pays que la commande a été reçue. ${ }^{116}$

En pratique, la loi applicable sera donc souvent celle du vendeur et l'on peut y voir un avantage pour lui, car elle est celle qu'il a le plus de facilités à connaître. De son côté, l'acheteur est défavorisé car il est en butte à un droit qui ne lui est pas familier. Ainsi, le droit international privé renverse le principe du Code civil, selon lequel le contrat doit s'interpréter contre le vendeur (art 1602).

En réalité, la Convention de La Haye de 1955 n'a pas connu un grand succès, comme en témoigne le faible nombre d'adhésion qu'elle a reçu. Et, d'ailleurs, une nouvelle Convention, sur la loi applicable à la vente internationale de marchandises, a été adoptée le 22 décembre 1986, qui a vocation à la remplacer. ${ }^{117}$

114 Et V. Civ. 1ère, 4 octobre 1989, Bull. civ. I, n 304, JCP. 1989. éd. E. I. 19179, J. dr. int. (Clunet) 1990. 415, note P. Kahn, Rev. crit. dr. int. priv. 1990. 316, note P. Lagarde, sacs de terreau vendus ayant provoqué a perte de plants, loi régissant la validité de clauses exonératoires de responsabilité: le juge doit rechercher la loi applicable au contrat, et en matière de vente appliquer d'office la Convention de La Haye de 1955; Com. 4 juin 1991, Bull. civ. IV, $\mathrm{n}^{\circ}$ 205, articles de maroquinerie, défaut de conformité, cassation de l'arrêt n'ayant pas recherché la loi applicable à la vente.

Selon les tribunaux, la Convention de La Haye n'est pas applicable aux contrats de concession, contrat-cadre distinct des ventes successives espérées entre fournisseur et distributeur, V. Civ. 1ère, 22 juillet 1986, Rev. crit. dr. int. priv. 1988. 57, note $\mathrm{H}$. Batiffol.

En ce qui concerne l'action d'un sous-acquéreur contre un vendeur antérieur d'une chose, qui est considérée en droit français comme étant purement contractuelle, V. Paris 14 juin 1989, D. 1989. IR. 201, qui admet l'application de la Convention de La Haye à uṇe telle hypothèse.

115 La désignation de la loi applicable par les parties doit faire l'objet d'une clause expresse ou résulter indubitablement du contrat, art. 2.

116 Plus précisément la loi du pays où l'acheteur a sa résidence habituelle, où dans lequel il possède l'établissement qui a passé la commande; par ailleurs, le texte précise qu'il s'agit de la loi "interne" du pays en cause, afin d'éviter tout phénomène de "renvoi", art. 3; et V. dans une hypothèse de conflit de qualifications, Civ. 1ère, 18 octobre 1989, Bull. civ. I, $n^{\circ} 322$, Rev. crit. dr. int. priv. 1990. 712, note Jacques Foyer, installation de séchage défectueuse, application du droit allemand au contrat, exclusion du droit français pour la responsabilité délictuelle.

117 V. Y. Loussouarn, La Convention de La Haye d'octobre 1985 sur la loi applicable aux contrats de vente de marchandises, Rev. crit. dr. int. priv. 1986. 271; V. Heuzé, La vente internationale, $n^{\circ} 33 \mathrm{~s}$. Le nouveau texte de La Haye répond au besoin de moderniser le dispositif, d'une part, en le coordonnant avec la Convention de Vienne, et, d'autre part, en prenant en compte des préoccupations nouvelles comme la protection des 


\section{Autres textes. Clauses du contrat}

En l'état actuel des choses, il est intéressant de se demander ce qui se passe lorsque se trouve saisi le juge d'un pays qui n'est pas partie à la Convention de 1955. Deux situations sont susceptibles de se présenter. Premièrement, il peut s'agir d'un Etat membre de la Communauté européenne, ayant ratifié la Convention de Rome du 19 juin 1980 sur la loi applicable aux obligations contractuelles, entrée en vigueur en 1991, et qui, sans être spécifique à la vente de marchandises, n'en couvre pas moins ce contrat. ${ }^{118}$ En fait, les règles posées par ce texte sont assez proches, dans les conséquences à en tirer pour la vente, de celles retenues par la Convention de La Haye, même si le raisonnement et la formulation retenue diffèrent: il se réfère à la loi choisie par les parties, ${ }^{119}$ à défaut de quoi est compétente la loi du pays avec lequel le contrat entretient les liens les plus étroits, une présomption étant posée que ce lien existe avec le pays où la partie qui doit fournir la prestation caractéristique a sa résidence habituelle ou, s'agissant d'un professionnel, son principal établissement. ${ }^{120}$ Or, en ce qui concerne la vente, c'est la loi du pays du vendeur que cela désigne.

Deuxièmement, le tribunal saisi peut être celui d'un pays qui n'est membre ni de la Convention de La Haye, ni de la Convention de Rome: alors est applicable, de la manière la plus classique, le droit international privé national propre à cet Etat. Tout au plus observera-t-on qu'un grand nombre de pays reconnaissent le principe de la loi d'autonomie, c'est à dire la faculté pour les parties de désigner la loi applicable au contrat qu'elles passent, et qu'en l'absence d'un tel choix ils font appel à des critères objectifs variés. ${ }^{121}$

Dans une large mesure, en tout cas, les règles de conflit de loi sont ainsi tributaires du juge saisi. ${ }^{122}$

consommateurs. A ce jour, toutefois, il n'a guère fait l'objet de ratifications, lui non plus: seule l'Argentine y a adhéré en 1991.

118 Sur l'entrée en vigueur de ce texte, V. H. Gaudemet-Tallon, Rev. trim. dr. eur. 1991. 635; Jacques Foyer, Entrée en vigueur de la Convention de Rome du 19 juin 1980 sur la loi applicable aux obligations contractuelles, J. dr. int. (Clunet) 1991. 601; adde, sur son contenu, P. Lagarde, Le nouveau droit international privé des contrats après l'entrée en vigueur de la Convention de Rome du 19 juin 1980, Rev. crit. dr. int. priv. 1991. 287 ('auteur était rapporteur officiel de la Convention).

119 Choix exprès, ou résultant de façon certaine des dispositions du contrat, ou des circonstances, art. 1er.

120 Art. 4-1 et 2; pour les contrats conclus avec des consommateurs, l'art. 5 prévoit que le choix de la loi applicable dans le contrat ne peut pas, en principe, priver le consommateur de la protection que lui accorde la loi de son pays de résidence.

121 V. V. Heuzé, La vente internationale, $\mathrm{n}^{\circ} 11 \mathrm{~s}$.

122 Pour les pays membres de la Communauté économique européenne, la Convention de Bruxelles du 27 septembre 1968 sur la compétence et l'effet des jugements, entrée en vigueur en 1973, a établi un espace judiciaire européen où les règles de conflit de juridiction sont uniformisées: elle pose le principe de la compétence 
D'où l'intérêt des clauses déterminant le droit applicable. Et celui qui s'attache à désigner la juridiction compétente, qui peut être un tribunal arbitral. Les parties ont une grande liberté en la matière, même à l'égard de la Convention Vienne. Elles peuvent en écarter l'application, alors même qu'elle serait compétente, et choisir un droit interne à l'exclusion des règles issues de la Convention que ce dernier pourrait contenir. ${ }^{123}$ Tout à l'inverse, quand bien même la Convention de Vienne serait inapplicable en principe à leur relations, elles ont la faculté de déclarer s'y soumettre, par exemple en choisissant la loi d'un pays qui l'a ratifiée.

Enfin, il faut mentionner l'existence de la Convention de La Haye du 2 octobre 1973, sur la loi applicable à la responsabilité du fait des produits, également ratifiée par la France, ${ }^{124}$ qui ouvre une option à trois branches en retenant la compétence de la loi de l'Etat de la résidence habituelle de la victime, de celle de l'Etat de l'établissement principal du responsable recherché, et de celle de l'Etat sur le territoire duquel a été acquis le produit. ${ }^{125}$

Malgré les efforts d'uniformisation, et l'existence d'instruments conventionnels de qualité, le bilan à dresser apparaît finalement assez maigre sur le terrain des conflits de lois, étant donné le peu de ratification que les textes ont jusqu'à ce jour connu.

du demandeur, le tribunal du lieu où l'obligation servant de base à l'action a été, ou doit être, exécutée (art. 5$1^{\circ}$ ), le tout sous réserve d'une clause attributive de juridiction convenue entre les parties, et dont la Convention admet la validité (art. 17); et V. Y. Loussouarn et $\mathrm{P}$. Bourel, $\mathrm{n}^{\circ} 484 \mathrm{~s} ; \mathrm{P}$. Mayer, $\mathrm{n}^{\circ} 339 \mathrm{~s}$.

Un texte distinct, la Convention de Lugano de 1988, étend les principes de la Convention de Bruxelles dans les rapports entre les Etats membres de la Communauté européenne et ceux de l'AELE.

En dehors de ce cadre, sont applicables les règles classiques des conflits de juridiction, qui dépendent du droit du tribunal saisi: elles font une large place à la compétence du tribunal du défendeur.

123 V. notamment C. Witz, L'exclusion de la Convention des Nations-Unies sur la vente internationale de marchandises par la volonté des parties, D. 1990. Chron. 107; Ph. Kahn, Choisir la Convention plutôt que le droit français, in "Le nouveau droit de la vente internationale", 1987, p $1253 \mathrm{~s}$.

124 Publiée par un décret du 10 octobre 1977, JO du 3 novembre 1977; les autres pays l'ayant ratifiée sont l'Espagne, le Luxembourg, la Norvège, les Pays-Bas et la Yougoslavie, et V. V. Heuzé, La vente internationale, p 381 .

125 La Convention, toutefois, vise exclusivement certaines d'hypothèses de responsabilité comme entrant dans son domaine, notamment celle où est recherchée la responsabilité du fabricant ou du fournisseur du produit (art. 3), et se déclare inapplicable en ce qui concerne les rapports - purement contractuels, apparemment - entre la victime et celui dont elle tient la propriété du produit, c'est à dire le contrat de vente au consommateur (art. 1er); par ailleurs, ici encore, c'est la loi "interne" désignée qui est déclarée compétente, de manière à écarter toute idée de "renvoi" par celle-ci à une loi tierce. 


\section{Bibliographie}

B. Audit, Droit international privé, Economica, 1991, notamment $\mathrm{n}^{\circ}$ 792; Batiffol et Lagarde, Droit international privé, T. 2, 1981, n 57 s; A. Bénabent, Contrats spéciaux, précis Domat, 1993, n 15; F. Collart Dutilleul et Ph. Delebecque, Contrats civils et commerciaux, précis Dalloz, 1993, n 106 s, 205 s, 231, 322 s, 343; J. Ghestin et B. Desché, Traité des contrats, La vente, LGDJ, 1990, $\mathrm{n}^{\circ} 1044 \mathrm{~s}$; J. Hamel, G. Lagarde et $\mathrm{H}$. Jauffret, Traité de droit commercial, Dalloz, T. 1, 2ème éd. 1980, 1er vol. Introduction, $\mathrm{n}^{\circ} 43 \mathrm{~s}$, et T. 2, 1966, $\mathrm{n}^{\circ} 1815 \mathrm{~s}$; R. Houin et M. Pédamon, Droit commercial, commerçants 1990, $n^{\circ} 563$ s; J. Huet, Responsabilité du vendeur et garantie contre les vices cachés, Litec, 1987; Y. Loussouarn et J.-D. Bredin, Droit du commerce international, Sirey $1969, \mathrm{n}^{\circ} 559 \mathrm{~s}$, et spé. $n^{\circ} 585 \mathrm{~s}$ (le droit unifié de la vente internationale); Y. Loussouarn et $\mathrm{P}$. Bourel, Droit international privé, précis Dalloz, 3ème éd. 1988, n 143 et 378; Ph. Malaurie et L. Aynès, Les contrats spéciaux, éd. Cujas 1992, n 65, 272, 286; P. Mayer, Droit international privé, précis Domat, 4ème éd. 1991, notamment n $640 \mathrm{~s}$ et $708 \mathrm{~s}$; G. Ripert et R. Roblot, Traité de droit commercial, T. 2, 11ème éd. LGDJ 1992 (4ème partie, Contrats commerciaux), $\mathrm{n}^{\circ} 2544$ s; Ripert et Boulanger, Traité de droit civil, T. 3, LGDJ 1958, n 1568 et 1600 s; J.-L. Rives-Lange et M. ContamineRaynaud, Droit bancaire, précis Dalloz, 1990, n 710 s (crédit documentaire); R. Rodière et E. du Pontavice, Droit maritime, précis Dalloz, 1991, n $420 \mathrm{~s}$.

Monographies: Ph. Kahn, La vente commerciale internationale, thèse, Paris, 1961; P. Malinverni, Les conditions générales de vente et les contrats-types des chambres syndicales, Thèse, L.G.D.J. 1978; J. Honnold, Uniform law for international sales, Kluwer 1982; J. Thieffry et C. Granier, La vente internationale, Collection de l'exportateur, CFCE, 1985; B. Audit, La vente internationale de marchandises (Convention des Nations-Unies du 11 avril 1980), LGDJ, 1990; V. Heuzé, La vente internationale de marchandises, JLN Joly, 1992; adde, M. Alter, L'obligation de délivrance dans la vente de meubles corporels, thèse, LGDJ 1972; J.-C. Boulay, La conformité des biens dans la vente de meubles corporels, Etude comparative, thèse Paris II, 1979, dactyl..

Colloques, ouvrages collectifs: "Les ventes internationales de marchandises", colloque de la fondation internationale pour l'enseignement du droit des affaires, Aix 1980, avant propos Y. Guyon, Economica 1981, avec les présentations de E. E. Bergsten, The law of sales in comparative law, p $3 \mathrm{~s}, \mathrm{M}$. Borysewicz, Conventions et projets de conventions sur la vente internationale, p $16 \mathrm{~s}, \mathrm{P}$. Bonassies, Vente internationale et droit de la concurrence, p $62 \mathrm{~s}$, A. Meinertzhagen-Limpens, Typologie des conditions générales dans la vente internationale d'objets mobiliers corporels, p 79 et s, M. R. Will, Conflits entre conditions générales de vente, p 99 s, J. Thieffry, La rédaction des conditions générales de vente, p 110 s, B. Stauder, Conformité et garanties (droit allemand), p 123 s, B. Stauder et H. Stauder, Conformité et garanties (droit suisse), p 161, P. A. Foirier, Conformité et garanties (droit belge), p 198, J. Darby, Le droit des Etats-Unis applicable à la vente internationale d'objets mobiliers corporels, $\mathrm{p}$ $223 \mathrm{~s}$, Ph. le Tourneau, Conformité est garanties (droit français), p $232 \mathrm{~s}, \mathrm{C}$. M. Schmittoff, Ventes internationales d'objets mobiliers corporels (Royaume-Uni), p 290 s, C. Ducouloux-Favard, Conformité et garanties (droit italien), p 298 s, E. Hondius, Conformité et garanties (droit néerlandais), p 312 s, N. N. Antzki, Les défauts de conformité et les vices cachés (droit québécois), p 340 s, J. Ghestin, Harmonisation des droits nationaux en matière de conformité et de garanties, p 369 s, X. Tandeau de Marsac, Le paiement et ses garanties, p 391 s, Conclusions par A. Tunc, p 407 s; "La Convention Vienne de 1980 sur la vente internationale de marchandises", Institut suisse de droit comparé (colloque de Lausanne des 19 et 20 novembre 1984), Zurich 1985; C. M. Bianca et M. J. Bonell, Commentary on the 
international sales law, Guiffré, Milan 1987, avec la collaboration de J. Barrera Graf, H. T. Bennett, S. K. Date-Bash, G. Eörsi, M. Evans, E. A. Farnsworth, E. Jayme, W. Khoo, V. Knapp, O. Lando, D. Maskow, B. Nicholas, J. Rajski, K. Sono, D. Tallon, et M. Will; "Le nouveau droit de la vente internationale", Colloque du 21 octobre 1987, CFCE, Cahiers juridiques et fiscaux, 1988, avec les présentations de E. E. Bergsten, Le rôle de la Convention des NationsUnies, p 1245 s, Ph. Kahn, Choisir la Convention plutôt que le droit français, p 1253 s, P. Thieffry, Le choix préalable du droit applicable, p $1265 \mathrm{~s}$; "La Convention de Vienne sur la vente internationale et les Incoterms", éd. Y. Derains et J. Ghestin, Actes du colloque du centre de droit des obligations de l'Université de Paris I, 1er et 2 décembre 1989, L.G.D.J. 1990, présentation par B. Audit, avec les interventions de D. Le Masson et S. Stenay, Les Incoterms, p 37 s, Ch. Mouly, La formation du contrat, p 57 s, J. Ghestin, Les obligations du vendeur, p 85 s, E. Robine, Difficultés d'application, p 119 s, Y. Derains, Transfert des risques de livraison, p 129 s, G. Flécheux, Les obligations de l'acheteur, p $141 \mathrm{~s}$, rapport de synthèse par ph. Fouchard, p $151 \mathrm{~s}$.

Articles: Ph. Kahn, La convention de Vienne du 11 avril 1980 sur les contrats de vente internationale de marchandise, Rev. int. de dr. comp. 1981. 95; J.-P. Plantard, Le nouveau droit uniforme de la vente internationale, La Convention des Nations-Unies du 11 avril 1980, J. dr. int. (Clunet) 1988. 339 (M. Plantard dirigeait la délégation française à la Conférence de Vienne et 1980); Ch. Mouly, Que change la Convention de Vienne sur la vente internationale par rapport au droit français interne?, D. 1991, Chron. p 77 à 79; B. Nicholas, The Vienna Convention on international sales law, The Law Quarterly Rev. Vol. 105, p 201 s (Apr. 1989), Prerequisites and extent of liability for breach of contract under the U.N. Convention, Einheitliches Kaufrecht und nationales Obligationenrecht, Baden-Baden 1987; Cl. Witz, L'exclusion de la Convention des Nations-Unies sur les contrats de vente internationale de marchandises par la volonté des parties, D. 1990. Chron. 107.

Encyclopédies: Ph. Kahn, J.-Cl. Dr. int., Fasc. 565-A-5, Vente commerciale internationale, 1989; J. Stoufflet, Rep. com. Dalloz, $V^{\circ}$ Crédit documentaire, 1989; Lamy, Contrats internationaux, sous la dir. de H. Lesguillons, T. 3, La vente; Dictionnaire Joly, Pratique des contrats internationaux, sous la direction de V. Heuzé.

Pour le droit comparé, V. notamment, Zweigert et Kötz, An introduction to comparative law, 1987, trad. Tony Weir, Vol. 2, relatif aux contrats; J. Thieffry et C. Granier, La vente internationale, Collection de l'exportateur, CFCE 1985, précité; sur le droit anglais de la vente, résultant actuellement du Sale of Goods Act de 1977, V. Droit anglais, sous la dir. de J. A. Jolowicz, précis Dalloz, 1986, spé. $\mathrm{n}^{\circ} 232 \mathrm{~s}$, le droit commercial par M. Clarke; sur le droit américain, c'est à dire le UCC-Sales, V. J. M. Stockton, "Sales", Coll. West In a nutschell, 1981; B. Stone, "Uniform commercial code", Coll. West In a nutschell, 1989; J. J. White et R. S. Summers, "Uniform commercial code", West 1988; Droit des Etats-Unis, précis Dalloz, sous la dir. de A. Levasseur, 1988, $\mathrm{n}^{\circ} 425 \mathrm{~s}$, le code de commerce uniforme par W. D. Hawkland; et pour connaître le texte en anglais du Sales of Goods Act de 1977, et du l'art 2 du UCC, ou UCC-Sales, V. H. Lesguillons, Droit des contrats internationaux, Lamy, $t$. 3, La vente; et, pour une traduction en français du UCC, Art. 1 et 2, V. C. Lombrecht, Le code de commerce uniforme américain, A. Collin, 1971. 


\section{Introduction to the International Sale of Goods}

The international sale of goods is a commercial sale in pure form. The operation is most frequently settled between traders, and the distance which separates them requires that it generally be accompanied by transport arrangements. This transport need poses special risks for the goods. The distance which separates the parties also complicates questions relating to the payment of the price. The rules provided in respect of domestic sales are illadapted to international sales.

The commercial and international dimension of the operation therefore justifies the adaptation of the common law rules of contract at the domestic level. The objective must be to endow the international sale of goods with rules which are not only adapted to it, but which also can be admitted at the international level. Only rules of homogeneous law, given the diverse commercial poles of the planet, can give international trade the desired legal security. This is the reason for the work undertaken for many years, by professional organisations or within the contexts of institutions such as the International Chamber of Commerce, to identify appropriate rules. The Vienna Convention which has been ratified by France has established since 1988 (the date of its entry into force in France) an entire body of law on the international sale of goods alongside the rules of the Civil Code applicable to domestic sales.

In this introduction to the law of international sales, the detail of the rules given to these contracts by the Vienna Convention of 1980 is not addressed. The focus here is on the role of practice in the uniformising of law relating to international sale of goods where, under the influence of professional bodies, efforts have been made to bring greater security into the relationship between the parties. This has been achieved in diverse ways: the preparation of standardised contract documents, the standardising of terminology (known as Incoterms) to define the links between the sale of goods and their transportation, the adoption of rules for documentary credits in order to ensure in the most favourable of conditions the payment of the price.

The paper also describes the efforts made to put in place a body of substantive law for international sales which is acceptable at the world level, and this has been done by the adoption of the Vienna Convention of 1980. Finally, a description is given of the operation of the conflict of laws rules which serve to determine which law is applicable to the contract in those cases where the solution to legal difficulties still depends upon the application of private international law principles. 